ANALYSIS \& PDE Volume $5 \quad$ No. $2 \quad 2012$

NALINI ANANTHARAMAN AND GABRIEL RIVIÈRE

DISPERSION AND CONTROLLABILITY FOR THE SCHRÖDINGER EQUATION ON NEGATIVELY CURVED MANIFOLDS 


\title{
DISPERSION AND CONTROLLABILITY FOR THE SCHRÖDINGER EQUATION ON NEGATIVELY CURVED MANIFOLDS
}

\author{
NAlini ANANTHARAMAN AND GABRIEL RivièRE
}

\begin{abstract}
We study the time-dependent Schrödinger equation $\imath \frac{\partial u}{\partial t}=-\frac{1}{2} \Delta u$, on a compact Riemannian manifold on which the geodesic flow has the Anosov property. Using the notion of semiclassical measures, we prove various results related to the dispersive properties of the Schrödinger propagator, and to controllability.
\end{abstract}

\section{Introduction}

Let $M$ be a smooth compact Riemannian manifold of dimension $d$ (without boundary). We denote by $\Delta$ the Laplacian on $M$. We are interested in understanding the regularizing properties of the Schrödinger equation

$$
\left.\iota \frac{\partial u}{\partial t}=-\frac{1}{2} \Delta u, \quad \text { where } u\right\rceil_{t=0} \in L^{2}(M) .
$$

More precisely, given a sequence of initial conditions $u_{n} \in L^{2}(M)$, we investigate the asymptotic behavior of the family

$$
v_{n}(d x)=\left(\int_{0}^{T}\left|e^{l t \Delta / 2} u_{n}(x)\right|^{2} d t\right) d \operatorname{Vol}(x)
$$

of measures (where Vol denotes the Riemannian volume measure on $M$ ).

We want to relate this question to the behavior of the geodesic flow, using results on propagation of singularities. For that purpose, we reformulate the question using the semiclassical formalism, and more specifically the notion of semiclassical measures. We consider a sequence of states $\left(u_{\hbar}\right)_{\hbar \rightarrow 0^{+}}$normalized in $L^{2}(M)$ (indexed by a parameter $\hbar>0$ going to 0 , which plays the role of Planck's constant in quantum mechanics), and for every $t \in \mathbb{R}$ we define the following family of distributions on the cotangent bundle $T^{*} M$ :

$$
\mu_{\hbar}(t)(a)=\int_{T^{*} M} a(x, \xi) d \mu_{\hbar}(x, \xi):=\left\langle e^{l t \Delta / 2} u_{\hbar}\left|\mathrm{Op}_{\hbar}(a)\right| e^{i t \Delta / 2} u_{\hbar}\right\rangle_{L^{2}(M)} \quad \text { for all } a \in \mathscr{C}_{o}^{\infty}\left(T^{*} M\right),
$$

where $\mathrm{Op}_{\hbar}(a)$ is a $\hbar$-pseudodifferential operator of principal symbol $a$ (see [Dimassi and Sjöstrand 1999], or Appendix A for a brief reminder). This construction gives a description of a state in terms of position and impulsion variables. Throughout the paper, we will denote by $U^{t}:=e^{l t \Delta / 2}$ the quantum propagator.

N. Anantharaman wishes to acknowledge the support of Agence Nationale de la Recherche, under the grant ANR-09-JCJC0099-01.

MSC2000: 35B37.

Keywords: Schrödinger equation, semiclassical analysis, control theory. 
By standard estimates on the norm of $\mathrm{Op}_{\hbar}(a)$ (the Calderón-Vaillancourt theorem), the map $t \mapsto \mu_{\hbar}(t)$ belongs to $L^{\infty}\left(\mathbb{R} ; \mathscr{D}^{\prime}\left(T^{*} M\right)\right)$, and is uniformly bounded in that space as $\hbar \rightarrow 0^{+}$. Thus, one can extract subsequences that converge in the weak-* topology of $L^{\infty}\left(\mathbb{R} ; \mathscr{D}^{\prime}\left(T^{*} M\right)\right)$. In other words, after possibly extracting a subsequence, we have

$$
\mu_{\hbar}(\theta \otimes a):=\int_{\mathbb{R}} \theta(t) a(x, \xi) \mu_{\hbar}(t)(d x, d \xi) d t \underset{\hbar \rightarrow 0}{\longrightarrow} \int_{\mathbb{R}} \theta(t) a(x, \xi) \mu(t)(d x, d \xi) d t
$$

for all $\theta \in L^{1}(\mathbb{R})$ and $a \in \mathscr{C}_{o}^{\infty}\left(T^{*} M\right)$. The main example to keep in mind is the case when $\theta$ is the characteristic function of some interval $[0, T]$. In that case we can write

$$
\mu_{\hbar}(\theta \otimes a)=\int_{0}^{T}\left\langle e^{l t \Delta / 2} u_{\hbar}\left|\mathrm{Op}_{\hbar}(a)\right| e^{l t \Delta / 2} u_{\hbar}\right\rangle d t=\hbar \int_{0}^{T / \hbar}\left\langle e^{l \tau \hbar \Delta / 2} u_{\hbar}\left|\mathrm{Op}_{\hbar}(a)\right| e^{l \tau \hbar \Delta / 2} u_{\hbar}\right\rangle d \tau
$$

In the last term we used the change of variable $t=\hbar \tau$ to express everything in terms of the flow $e^{l \tau \hbar \Delta / 2}$, which solves the equation $-\hbar^{2} \Delta v / 2=\imath \hbar \partial v / \partial \tau$ with the time-parametrization of quantum mechanics. Thus, in the time-scale of quantum mechanics, we are averaging over time intervals of order $\hbar^{-1}$.

It follows from standard properties of $\mathrm{Op}_{\hbar}(a)$ that the limit $\mu$ has the following properties:

- For almost all $t, \mu(t)$ is a positive measure on $T^{*} M$.

- The unitarity of $U^{t}$ implies that $\int_{T^{*} M} \mu(t)(d x, d \xi)$ does not depend on $t$; from the normalization of $u_{\hbar}$, we have $\int_{T^{*} M} \mu(t)(d x, d \xi) \leq 1$, the inequality coming from the fact that $T^{*} M$ is not compact, and that there may be an escape of mass to infinity.

- Define the geodesic flow $g^{\tau}: T^{*} M \rightarrow T^{*} M$ as the Hamiltonian flow associated with the energy $p(x, \xi)=\|\xi\|_{x}^{2} / 2$. From the Egorov theorem, we have

$$
e^{-i \tau \hbar \Delta / 2} \mathrm{Op}_{\hbar}(a) e^{i \tau \hbar \Delta / 2}=\mathrm{Op}_{h}\left(a \circ g^{\tau}\right)+O_{\tau, a}(\hbar) \text { for all } \tau \in \mathbb{R}
$$

and for $a \in \mathscr{C}_{o}^{\infty}\left(T^{*} M\right)$. At the limit $\hbar \rightarrow 0^{+}$, this implies that $\mu(t)$ is invariant under $g^{\tau}$ for almost all $t$ and all $\tau$.

These sequences of distributions were already studied by Macià [2009]; we refer to that paper for details about the facts mentioned above. Macià was mostly interested in describing the properties of the measures $\mu(t)$ in the case where the geodesic flow on the manifold $M$ was not chaotic (Zoll manifolds for instance, or the flat torus [Macià 2010; Anantharaman and Macià 2011]).

In this paper, we are interested in a completely different situation where the geodesic flow has the Anosov property (manifolds of negative curvature are the main example). In this setting, the case where the initial states $u_{\hbar}$ are eigenfunctions of the Laplacian, satisfying $-\hbar^{2} \Delta u_{\hbar}=u_{\hbar}$, has been much studied; in this particular situation $\mu_{\hbar}(t)$ does not depend on $t$. The Shnirelman theorem (also called quantum ergodicity theorem) says that for a "typical" sequence of eigenfunctions $u_{\hbar}$, the limit $\mu$ is the Liouville measure on the unit cotangent bundle $S^{*} M$; see [Shnirelman 1974; Zelditch 1987; Colin de Verdière 1985] for the precise statement. It is also known, by the work of Anantharaman and Nonnenmacher, that for any sequence of eigenfunctions the limit $\mu$ has positive entropy [Anantharaman 2008; Anantharaman and Nonnenmacher 2007; Anantharaman et al. 2009]. The aim of this paper is twofold: extend the 
Shnirelman theorem to the setting of the time dependent equation and prove lower bounds on the metric entropy of the measures $\mu(t)$. We shall also show how these results apply to the controllability problem for the Schrödinger equation.

\section{Statement of the results}

2a. Semiclassical large deviations. Our first result is a generalization (and a reinforcement in the case of Anosov geodesic flows) of the quantum ergodicity theorem. Recall that the Shnirelman theorem is originally a result on orthonormal bases of eigenfunctions of the Laplacian. In order to state an analogue for solutions of the time dependent Schrödinger equation, we introduce a notion of generalized orthonormal families.

2a1. Generalized orthonormal family. We fix $\alpha>0$ and a sequence $I(\hbar):=[a(\hbar), b(\hbar)]$ of subintervals that are of length at least $2 \alpha \hbar$ for every $\hbar>0$. We also assume that $\lim _{\hbar \rightarrow 0^{+}} a(\hbar)=\lim _{\hbar \rightarrow 0^{+}} b(\hbar)=1$. We denote by $N(I(\hbar))$ the number of eigenvalues $\lambda_{j}^{2}$ of $\Delta$ (counted with their multiplicities) satisfying $\hbar^{2} \lambda_{j}^{2} \in I(\hbar)$. We assume that

$$
N(I(\hbar))=\frac{\operatorname{Vol}(M)}{(2 \pi \hbar)^{d}} \operatorname{Vol}\left(B_{d}(0,1)\right)(b(\hbar)-a(\hbar))(1+o(1))
$$

(where $\operatorname{Vol}(M)$ is the Riemannian volume of $M$, and $\operatorname{Vol}\left(B_{d}(0,1)\right)$ is the volume of the unit ball in $\mathbb{R}^{d}$ ). According to [Duistermaat and Guillemin 1975], we know that the Weyl law (4) holds in the case where $b(\hbar)-a(\hbar)=2 \alpha \hbar$ if we suppose that the set of closed geodesics is of zero Liouville measure on $S^{*} M$ (this is the case for Anosov geodesic flows).

We introduce the notion of generalized orthonormal family localized in the "energy window" $I(\hbar)$ :

Definition 2.1. For $\hbar>0$, let $\left(\Omega_{\hbar}, \mathbb{P}_{\hbar}\right)$ be a probability space and $u_{\hbar}: \Omega_{\hbar} \rightarrow L^{2}(M)$ a measurable map. We say that $\left(u_{\hbar}(\omega)\right)_{\omega \in\left(\Omega_{h}, \mathbb{P}_{h}\right)}$ is a generalized orthonormal family (GOF) in the spectral window $I(\hbar)$ if

- $\left\|u_{\hbar}(\omega)\right\|_{L^{2}(M)}=1+o(1)$ as $\hbar$ tends to 0 (uniformly for $\omega$ in $\Omega_{\hbar}$ );

- $\left\|\left(\operatorname{Id}_{L^{2}(M)}-\mathbb{1}_{I(\hbar)}\left(-\hbar^{2} \Delta\right)\right) u_{\hbar}(\omega)\right\|_{L^{2}(M)}=o(1)$ as $\hbar$ tends to 0 (uniformly for $\omega$ in $\Omega_{\hbar}$ );

- for every $B$ in $\mathscr{L}\left(L^{2}(M)\right)$,

$$
\int_{\Omega_{\hbar}}\left\langle u_{\hbar}(\omega)|B| u_{\hbar}(\omega)\right\rangle_{L^{2}(M)} d \mathbb{P}_{\hbar}(\omega)=\frac{1}{N(I(\hbar))} \operatorname{Tr}\left(B \mathbb{1}_{I(\hbar)}\left(-\hbar^{2} \Delta\right)\right) .
$$

We stress the fact that if $\left(u_{\hbar}(\omega)\right)_{\omega \in\left(\Omega_{h}, \mathbb{P}_{h}\right)}$ is a GOF, then $\left(U^{t} u_{\hbar}(\omega)\right)_{\omega \in\left(\Omega_{h}, \mathbb{P}_{h}\right)}$ is also one for every $t$. This is a strong requirement which is crucial in the sequel. In Section 4, we will provide two examples of GOF.

We will denote by $\mu_{\hbar, \omega}(t)$ the (time-dependent) distribution associated to $u_{\hbar}(\omega)$ by formula (2).

2a2. Semiclassical large deviations. The quantum ergodicity theorem says that, for a given orthonormal basis of eigenvectors of $\Delta$, "most of" the associated distributions on $T^{*} M$ converge to the Liouville measure on the unit cotangent bundle $S^{*} M:=\{p=1 / 2\}$ (we recall that $p(x, \xi)=\|\xi\|_{x}^{2} / 2$ is the classical energy). This holds under the assumption that the geodesic flow acts ergodically on $S^{*} M$ endowed with 
the Liouville measure. Here we aim for a more precise statement, and will assume that the geodesic flow has the Anosov property. Our result will, in particular, imply a reinforced version of the usual Shnirelman theorem.

We recall that the Liouville measure on $T^{*} M$ is the measure given by $d \mathscr{L}=d x d \xi$ in local coordinates. In a region where the Hamiltonian $p$ has no critical point, one can find local symplectic coordinates $\left(x_{1}, \ldots, x_{d}, \xi_{1}, \ldots, \xi_{d}\right)$ such that $x_{1}=p$, and the Liouville measure can be decomposed into $d \mathscr{L}=$ $d x_{1} d L_{x_{1}}(x, \xi)$, where $L_{x_{1}}$ is a smooth positive measure carried by the energy layer $\left\{p=x_{1}\right\}$. We shall restrict our attention to the unit cotangent bundle, $S^{*} M=\left\{p=\frac{1}{2}\right\}$, and will denote $L=L_{1 / 2}$. This is the Liouville measure on $S^{*} M$.

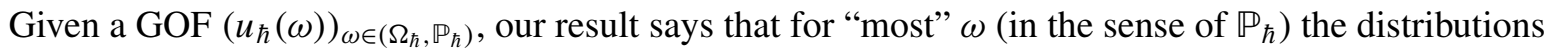
$\mu_{\hbar, \omega}(t)$ are close to the Liouville measure $L$. We will use a large deviations result due to Kifer [1992] to give an estimate on the proportion of $\omega$ for which $\mu_{\hbar, \omega}(t)$ is far away from $L$. To state our result, we need to introduce two dynamical quantities. First, we define the maximal expansion rate of the geodesic flow on $S^{*} M$ as

$$
\chi_{\max }:=\lim _{\tau \rightarrow \pm \infty} \frac{1}{\tau} \log \sup _{\rho \in S^{*} M}\left\|d_{\rho} g^{\tau}\right\| .
$$

This quantity gives an upper bound on the Lyapunov exponents over $S^{*} M$ and it is linked to the range of validity of the semiclassical approximation in the Egorov theorem [Bouzouina and Robert 2002]. We also introduce, for every $\delta$ in $\mathbb{R}$ and every $a$ in $\mathscr{C}_{o}^{\infty}\left(T^{*} M, \mathbb{R}\right)$ such that $L(a)=0$,

$$
H(\delta):=\inf _{s \in \mathbb{R}}\left\{-s \delta+P\left(s a+\varphi^{u}\right)\right\},
$$

where $f \mapsto P(f)$ is the topological pressure of the continuous map $f$ and $\varphi^{u}$ is the infinitesimal unstable Jacobian (see Section 3 for details). The map $\delta \mapsto-H(\delta)$ is the Legendre transform of $s \mapsto P\left(s a+\varphi^{u}\right)$, which is a smooth and convex function on $\mathbb{R}$. In particular, $-H$ is a convex map on $\mathbb{R}$ and it satisfies $H(0)=0$ and $H(\delta)<0$ for all $\delta \neq 0$ (see Section 3c).

Theorem 2.2. Suppose $\left(S^{*} M,\left(g^{\tau}\right)\right)$ has the Anosov property. We fix a sequence of generalized orthonormal families $\left(u_{\hbar}(\omega)\right)_{\omega \in\left(\Omega_{\hbar}, \mathbb{P}_{h}\right)}\left(\right.$ with $\left.\hbar \rightarrow 0^{+}\right)$. We fix two observables,

- an element $\theta$ in $L^{1}\left(\mathbb{R}, \mathbb{R}_{+}\right)$such that $\int \theta(t) d t=1$, and

- an element a in $\mathscr{C}_{o}^{\infty}\left(T^{*} M, \mathbb{R}\right)$ such that $\int_{S^{*} M}$ adL $=0$.

Then, we have, for any $\delta>0$,

$$
\limsup _{\hbar \rightarrow 0} \frac{\log \mathbb{P}_{\hbar}\left(\left\{\omega \in \Omega_{\hbar}: \mu_{\hbar, \omega}(\theta \otimes a) \geq \delta\right\}\right)}{|\log \hbar|} \leq \frac{H(\delta)}{\chi_{\max }} .
$$

From this theorem and the properties of $H(\delta)$, one can deduce the following corollary:

Corollary 2.3. Suppose $\left(S^{*} M,\left(g^{\tau}\right)\right)$ has the Anosov property. Fix a sequence of $G O F\left(u_{\hbar}(\omega)\right)_{\omega \in\left(\Omega_{h}, \mathbb{P}_{h}\right)}$ $\left(\right.$ with $\left.\hbar \rightarrow 0^{+}\right)$. Then, for every $\delta>0$, for every $a \in \mathscr{C}_{o}^{\infty}\left(T^{*} M, \mathbb{C}\right)$ and for every function $\theta$ in $L^{1}\left(\mathbb{R}, \mathbb{R}_{+}\right)$, we have

$$
\mathbb{P}_{\hbar}\left(\left\{\omega \in \Omega_{\hbar}:\left|\mu_{\hbar, \omega}(\theta \otimes a)-\int_{S^{*} M} a d L \int_{\mathbb{R}} \theta(t) d t\right| \geq \delta\right\}\right)=\mathbb{O}_{a, \delta, \theta}\left(\hbar^{\tilde{H}(\delta)}\right),
$$


where $\tilde{H}(\delta)>0$ depends on $a, \theta$ and $\delta$.

2a3. Comments. As already mentioned, this result reinforces the Shnirelman theorem in the case of Anosov geodesic flows. The Shnirelman theorem (suitably adapted to the time-dependent Schrödinger equation) would simply assert that for an ergodic geodesic flow, and for every $\delta>0$,

$$
\mathbb{P}_{\hbar}\left(\left\{\omega \in \Omega_{\hbar}:\left|\mu_{\hbar, \omega}(\theta \otimes a)-\int_{S^{*} M} a d L \int_{\mathbb{R}} \theta(t) d t\right| \geq \delta\right\}\right)=o_{a, \delta, \theta}(1) .
$$

The algebraic rate of Corollary 2.3 can be compared with a classical conjecture in quantum chaos, known as the quantum variance conjecture [Feingold and Peres 1986; Eckhardt et al. 1995]. This conjecture is usually formulated for eigenfunctions of the Laplacian and states that the quantum variance behaves (modulo a prefactor related to a classical variance) like $1 / T_{H}(\hbar)$, where $T_{H}(\hbar)$ is the Heisenberg time. Recall that the Heisenberg time is defined as $\hbar \bar{\rho}(\hbar)$, where $\bar{\rho}(\hbar)$ is the mean density of states (which is proportional to $\hbar^{-d}$ in our case). Translated in our context, it would predict that

$$
\int_{\Omega_{h}}\left|\mu_{\hbar, \omega}(\theta \otimes a)-\int_{S^{*} M} a d L \int_{\mathbb{R}} \theta(t) d t\right|^{2} d \mathbb{P}_{\hbar}(\omega) \sim V(a, \theta) \hbar^{d-1},
$$

where $V(a, \theta)$ would be a classical dynamical variance. If this conjecture is true, it implies

$$
\mathbb{P}_{\hbar}\left(\left\{\omega \in \Omega_{\hbar}:\left|\mu_{\hbar, \omega}(\theta \otimes a)-\int_{S^{*} M} a d L \int_{\mathbb{R}} \theta(t) d t\right| \geq \delta\right\}\right)=\mathcal{O}\left(\hbar^{d-1}\right),
$$

which is stronger than our result.

Related to this kind of question, Zelditch [1994] proved that

$$
\int_{\Omega_{\hbar}}\left|\mu_{\hbar, \omega}(\theta \otimes a)-\int_{S^{*} M} a d L \int_{\mathbb{R}} \theta(t) d t\right|^{p} d \mathbb{P}_{\hbar}(\omega)=\mathcal{O}\left(|\log \hbar|^{-p / 2}\right)
$$

for all $p \geq 1$; see also [Schubert 2006]. Again, his proof is written for the eigenfunction problem, but could easily be transposed to the time-dependent Schrödinger equation (see [Rivière 2009] — and note that we have to make the extra assumption $\left\|u_{\hbar}(\omega)\right\|_{L^{2}}=1+\mathcal{O}\left(|\log \hbar|^{-1}\right)$ uniformly in $\left.\omega\right)$. Using the Bienaymé-Chebyshev inequality, Zelditch's result implies that

$$
\mathbb{P}_{\hbar}\left(\left\{\omega \in \Omega_{\hbar}:\left|\mu_{\hbar, \omega}(\theta \otimes a)-\int_{S^{*} M} a d L \int_{\mathbb{R}} \theta(t) d t\right| \geq \delta\right\}\right)=\mathcal{O}\left(|\log \hbar|^{-\infty}\right) .
$$

Our theorem — although it does not say anything about the quantum variance — improves this aspect of Zelditch's result, as we can replace $\mathcal{O}\left(|\log \hbar|^{-\infty}\right)$ by $\mathcal{O}\left(\hbar^{\tilde{H}(\delta)}\right)$.

2b. Entropy of semiclassical measures. Our second result is a lower bound on the Kolmogorov-Sinai entropy of the measures $\mu(t)$. We will consider a sequence of normalized states $\left(u_{\hbar}\right)_{\hbar \rightarrow 0^{+}}$in $L^{2}(M)$. We fix two energy levels $0 \leq E_{1}<E_{2}$ and we suppose that the family of states is localized in the energy window $\left[E_{1}, E_{2}\right]$. Precisely, we make the assumption that

$$
\lim _{\hbar \rightarrow 0^{+}}\left\|\left(\operatorname{Id}_{L^{2}(M)}-\mathbb{1}_{\left[E_{1}, E_{2}\right]}\left(-\hbar^{2} \Delta\right)\right) u_{\hbar}\right\|_{L^{2}(M)}=0 .
$$


This assumption implies that each $\mu(t)$ is a probability measure carried by the set $\left\{E_{1} \leq\|\xi\|_{x}^{2} \leq E_{2}\right\}$ (it prevents escape of mass in the fibers of $T^{*} M$ ). In addition, we recall that $\mu(t)$ is invariant under the geodesic flow. Using the invariance of the energy under the geodesic flow, we see that for Lebesgue almost every $t, \mu(t)(d x, d \xi)$ is of the form $\int \mu_{t, E}(d x, d \xi) v(d E)$, where $v$ is a positive measure on the interval $\left[E_{1}, E_{2}\right]$ and $\mu_{t, E}$ is a probability measure on $\left\{\|\xi\|_{x}^{2}=E\right\}$ invariant under the geodesic flow.

Remark 1. The measure $v$ is independent of $t$. It is the weak limit (after extraction of a subsequence) of the measures $v_{\hbar}$ defined on $\mathbb{R}$ by $v_{\hbar}\left(\left[E, E^{\prime}\right]\right)=\left\|\mathbb{1}_{\left[E, E^{\prime}\right]}\left(-\hbar^{2} \Delta\right) u_{\hbar}\right\|^{2}$.

In the following theorem, $h_{\mathrm{KS}}\left(\mu,\left(g^{\tau}\right)\right)$ denotes the entropy of the invariant probability measure $\mu$ for the geodesic flow $g^{\tau}$ (its definition is recalled in Section 3).

Theorem 2.4. Let $M$ be a compact Riemannian manifold of dimension d and constant curvature $\equiv-1$. We fix two energy levels $0 \leq E_{1}<E_{2}$ and we consider a sequence $\left(u_{\hbar}\right)_{\hbar \rightarrow 0^{+}}$in $L^{2}(M)$ that satisfies

- the energy localization $\lim _{\hbar \rightarrow 0}\left\|\left(\operatorname{Id}_{L^{2}(M)}-\mathbb{1}_{\left[E_{1}, E_{2}\right]}\left(-\hbar^{2} \Delta\right)\right) u_{\hbar}\right\|_{L^{2}(M)}=0$ and

- $\lim _{\hbar \rightarrow 0}\left\|u_{\hbar}\right\|_{L^{2}(M)}=1$.

Consider $\mu(t)(d x, d \xi)=\int \mu_{t, E}(d x, d \xi) v(d E)$ a weak-* limit in $L^{\infty}\left(\mathbb{R} ; \mathscr{D}^{\prime}\left(T^{*} M\right)\right)$ of the sequence of distributions $\mu_{\hbar}(t)$ defined in (2). Then, one has, Leb $\otimes v$ almost everywhere,

$$
h_{\mathrm{KS}}\left(\mu_{t, E},\left(g^{\tau}\right)\right) \geq \frac{d-1}{2} \sqrt{E},
$$

where $h_{\mathrm{KS}}\left(\mu_{t, E},\left(g^{\tau}\right)\right)$ is the Kolmogorov-Sinai entropy of $\mu_{t, E}$.

Remark 2. For the sake of simplicity, we only state and prove the results in the case of constant curvature. In principle the methods from [Anantharaman and Nonnenmacher 2007; Anantharaman et al. 2009] for general Anosov manifolds or from [Rivière 2010] for Anosov surfaces could be adapted in this setting. However, one step requires a nontrivial adaptation: see Remark 8. Modulo this extra work, the result in variable curvature would read

$$
h_{\mathrm{KS}}\left(\mu_{t, E},\left(g^{\tau}\right)\right) \geq\left(\int\left|\varphi^{u}\right| d \mu_{t, E}-\frac{d-1}{2} \chi_{\max }(E)\right)
$$

where $\varphi^{u}$ is the unstable Jacobian and $\chi_{\max }(E)$ is the maximal expansion rate of the geodesic flow on the energy layer $\{p=E / 2\}$; see Section 3. This lower bound may be negative (and thus trivial) if $\chi_{\max }$ is too large compared to the average of $\varphi^{u}$. For surfaces, the adaptation of the ideas of [Rivière 2010] would lead to the better result

$$
h_{\mathrm{KS}}\left(\mu_{t, E},\left(g^{\tau}\right)\right) \geq \frac{1}{2} \int\left|\varphi^{u}\right| d \mu_{t, E}>0 .
$$

Remark 3. We note that $\sqrt{E}$ is the speed of trajectories of $g^{\tau}$ on the energy layer $\{p=E / 2\}$. It is also natural to consider the geodesic flow $\phi^{\tau}=g^{\tau / \sqrt{E}}$ parametrized to have speed 1 on any energy layer, and our result then reads $h_{\mathrm{KS}}\left(\mu_{t, E},\left(\phi^{\tau}\right)\right) \geq(d-1) / 2$.

If one wants, one can avoid assumption (7) and deal with the issue of escape of mass in a different manner: Consider the space $\mathscr{S}_{0}$ of smooth functions $a$ on $T^{*} M$ that are 0 -homogeneous outside a compact 
set. The distributions $\mu_{\hbar}(t)$ are bounded in $L^{\infty}\left(\mathbb{R}, \varphi_{0}^{\prime}\right)$, and one can consider convergent subsequences in the corresponding weak-* topology. The corresponding limits $\mu \in L^{\infty}\left(\mathbb{R}, \mathscr{S}_{0}^{\prime}\right)$ are actually positive for almost all $t$, and each $\mu(t)$ defines a probability measure on $\widehat{T^{*} M}$, the cotangent bundle compactified by spheres at infinity. We note that the flow $\phi^{t}$ can be extended to the spheres at infinity. We can then write $\mu(t)=\int \mu_{t, E}(d x, d \xi) v(d E)$, where now $v$ is a probability measure on $[0,+\infty]$. Our result reads $h_{\mathrm{KS}}\left(\mu_{t, E},\left(g^{\tau}\right)\right) \geq \sqrt{E}(d-1) / 2$ for $0 \leq E<+\infty$, and $h_{\mathrm{KS}}\left(\mu_{t, E},\left(\phi^{\tau}\right)\right) \geq(d-1) / 2$ for $0<E \leq+\infty$.

Remark $4\left(u_{\hbar}\right.$ versus $\left.u_{n}\right)$. Let $\left(u_{n}\right)$ be a normalized sequence in $L^{2}(M)$, and suppose we want to study the sequence of probability measures (1). No scale $\hbar_{n}$ is given a priori. We can always choose $\hbar_{n}$ such that (7) is satisfied, and apply Theorem 2.4. However, the statement of the theorem is trivial for the part of the limit measure carried on $\{\xi=0\}$ : It just says that $h_{\mathrm{KS}}\left(\mu_{t, 0}, g^{\tau}\right) \geq 0$. Thus, it is preferable to choose $\hbar_{n}$ such that none of the limit mass goes to $\{\xi=0\}$. If $u_{n}$ converges weakly to 0 in $L^{2}$, this is also possible but in general (7) will no longer be satisfied (some of the mass will escape to infinity) and one must in this case use the version of the theorem stated in Remark 3. If $u_{n}$ converges weakly to 0 in $L^{2}$ and if one is ready to have all the mass escape to infinity (thus losing some information about the rate of escape), one can even let $\hbar_{n}=1$. This means that one considers the "distribution"

$$
\mu_{n}(t)(b):=\left\langle u_{n} \mid e^{-l t \Delta / 2} \mathrm{Op}_{1}(b) e^{l t \Delta / 2} u_{n}\right\rangle_{L^{2}(M)},
$$

defined for all $b \in \mathscr{S}_{0}$. This is the analogue of (2) in the microlocal setting [Gérard 1991]. The map $t \mapsto \mu_{n}(t)$ belongs to $L^{\infty}\left(\mathbb{R}, \mathscr{Y}_{0}^{\prime}\right)$. Thus, there exists a subsequence $\left(u_{n_{k}}\right)_{k}$ and $\mu$ in $L^{\infty}\left(\mathbb{R}, \mathscr{S}_{0}^{\prime}\right)$ such that

$$
\int_{\mathbb{R} \times \widehat{T^{*} M}} \theta(t) b(x, \xi) \mu_{n_{k}}(t)(d x, d \xi) d t \underset{k \rightarrow+\infty}{\longrightarrow} \int_{\mathbb{R} \times \widehat{T^{*} M}} \theta(t) b(x, \xi) \mu(t)(d x, d \xi) d t
$$

for all $\theta \in L^{1}(\mathbb{R})$ and $b \in \mathscr{S}_{0}$. Besides, as above, $\mu(t)$ is a probability measure on the compactified cotangent bundle $\widehat{T^{*} M}$, and is invariant under the normalized geodesic flow. As $u_{n}(t)=e^{l t \Delta / 2} u_{n}$ converges weakly to 0 for every $t$ in $\mathbb{R}$, each $\mu(t)$ is actually supported at infinity, and may thus be identified with a probability measure on the unit sphere bundle $S^{*} M$, invariant under the geodesic flow.

Theorem 2.4 adapted to this setting says that $h_{\mathrm{KS}}\left(\mu(t),\left(g^{\tau}\right)\right) \geq(d-1) / 2$ for every $t$ in $\mathbb{R}$.

2c. Application to controllability. Theorem 2.4, in the form given in Remark 4, implies the following observability inequality:

Theorem 2.5. Let $M$ be a compact Riemannian manifold of dimension $d$ and constant curvature identically equal to -1 . Let a be a smooth function on $M$, and define a closed $g^{\tau}$-invariant subset of $S^{*} M$ by

$$
K_{a}=\left\{\rho \in S^{*} M, a^{2}\left(g^{\tau}(\rho)\right)=0 \text { for all } \tau \in \mathbb{R}\right\} .
$$

Assume that the topological entropy of $K_{a}$ is less than $(d-1) / 2$. Then, for all $T>0$, there exists $C_{T, a}>0$ such that, for all $u$ in $L^{2}(M)$,

$$
\|u\|_{L^{2}(M)}^{2} \leq C_{T, a} \int_{0}^{T}\left\|a e^{l t \Delta / 2} u\right\|_{L^{2}(M)}^{2} d t .
$$


Remark 5. The topological entropy of a $\left(g^{\tau}\right)$-invariant compact subset $K$ of $S^{*} M$ is related to the Kolmogorov-Sinai entropy by the variational principle [Walters 1982]

$$
h_{\text {top }}\left(K,\left(g^{\tau}\right)\right):=\sup _{\mu \in \mathcal{M}\left(S^{*} M, g^{\tau}\right)}\left\{h_{\mathrm{KS}}\left(\mu,\left(g^{\tau}\right)\right): \mu(K)=1\right\} \text {, }
$$

where $\mathcal{M}\left(S^{*} M, g^{\tau}\right)$ is the set of probability measures on $S^{*} M$ invariant under the geodesic flow. Thanks to [Barreira and Wolf 2007, Corollary 4], our assumption on the topological entropy of $K_{a}$ is satisfied when the Hausdorff dimension of $K_{a}$ is less than $d$. The converse is also true if $K_{a}$ is a locally maximal subset [Pesin and Sadovskaya 2001, Theorem 4.1], that is, there exists an open neighborhood $U$ of $K_{a}$ such that $K_{a}=\bigcap_{\tau \in \mathbb{R}} g^{\tau} \tilde{u}$.

The proof that Theorem 2.4 implies Theorem 2.5 is given in Section 7. This follows a classical argument due to Lebeau [1992], who used it to prove that if $M$ is an arbitrary Riemannian manifold, and if $K_{a}=\varnothing$ (the "geometric control condition"), then (8) holds.

We can give an example where our assumption on the topological entropy of $K_{a}$ is satisfied. Consider a closed geodesic $\gamma$ and a small tubular neighborhood of this geodesic in $M$ that does not contain another complete geodesic. We take $a$ to be nonzero on the complement of this neighborhood and 0 near the closed geodesic. In this case, one has $K_{a}=\gamma$ so that our condition holds. Another example, in dimension $d=2$, goes as follows: Take a decomposition of the hyperbolic surface $M$ into "hyperbolic pairs of pants" (there are $2 g-2$ pairs of pants if $M$ has genus $g$ ). The boundary of each pair of pants consists of 3 simple closed geodesics. Take a function $a$ supported in a neighborhood of the union of these $3 g-3$ simple closed geodesics, and assume that $a$ does not vanish on the union of these curves. Thus, any geodesic that avoids the support of $a$ must stay inside one of the pairs of pants. If the length of each of the $3 g-3$ boundary components is large enough, this will imply that $K_{a}$ has dimension less than $d$, and our condition will be satisfied. The existence of a hyperbolic pants decomposition with boundary components of arbitrary large lengths follows, for instance, from [Rees 1981, Proposition 2.2]. It would be interesting to find a larger variety of geometric situations in which our assumption on $K_{a}$ holds.

Following the Hilbert uniqueness method, one knows that inequality (8) implies that for any $u_{0}, u_{T} \in$ $L^{2}(M)$ and any $T>0$, there exists $f(t, x) \in L^{2}([0, T] \times M)$ such that the solutions of

$$
l \frac{\partial u}{\partial t}+\frac{\Delta}{2} u=a(x) f(t, x)
$$

with initial condition $\left.u\right|_{t=0}=u_{0}$ satisfy $\left.u\right|_{t=T}=u_{T}$. This is called the controllability problem.

Remark 6. As already mentioned, this application to the controllability problem relies on the entropic estimate of Theorem 2.4, which is proved for manifolds of constant negative curvature. In Remark 2, we indicated what should be (modulo extra work) the extension of Theorem 2.4 in the case of manifolds of variable negative curvature. Let us mention what would then be the consequences for controllability. In the case of manifolds of variable negative curvature, controllability would hold under the condition that

$$
P_{\text {top }}\left(K_{a},\left(g^{\tau}\right), \varphi^{u}\right)<-\frac{d-1}{2} \chi_{\max },
$$


where $P_{\text {top }}\left(K_{a},\left(g^{\tau}\right), \varphi^{u}\right)$ is the topological pressure of $K_{a}$ with respect to $\varphi^{u}$ [Pesin 1997, Appendix II]. If $M$ is of variable curvature, there is no precise relation between such a condition and the Hausdorff dimension of $K_{a}$. In the case of surfaces of variable negative curvature, the entropic estimate of Remark 2 would imply that controllability holds under the more general condition

$$
P_{\text {top }}\left(K_{a},\left(g^{\tau}\right), \frac{1}{2} \varphi^{u}\right)<0 .
$$

This condition is satisfied when the Hausdorff dimension of $K_{a}$ is less than 2 [Barreira and Wolf 2007, Corollary 4].

Organization of the paper. In Section 3, we describe some background in dynamical systems that we will need at different points of the article. In Section 4, we give two examples of GOF and apply Theorem 2.2 to them. In Sections 5 and 6, we prove Theorems 2.2 and 2.4. Finally, in Section 7, we show how to derive an observability result from Theorem 2.4. In the appendix, we give a brief review of semiclassical calculus on a manifold.

\section{Dynamical systems background}

3a. Anosov property. In this paper, we suppose that $M$ is a smooth, compact, Riemannian manifold of dimension $d$ (without boundary). The geodesic flow $\left(g^{\tau}\right)$ on $T^{*} M$ is the Hamiltonian flow associated to the Hamiltonian $p(x, \xi)=\|\xi\|_{x}^{2} / 2$. We also assume that, for any $E>0$, the geodesic flow $g^{\tau}$ is Anosov on the energy layer $p^{-1}(\{E / 2\}) \subset T^{*} M$ : For all $\rho \in p^{-1}(\{E / 2\})$, we have a decomposition

$$
T_{\rho} p^{-1}(\{E / 2\})=E^{u}(\rho) \oplus E^{s}(\rho) \oplus \mathbb{R} X_{p}(\rho),
$$

with $X_{p}$ is the Hamiltonian vector field associated to $p, E^{u}$ the unstable space and $E^{s}$ the stable space [Katok and Hasselblatt 1995]. We can introduce the infinitesimal unstable Jacobian as follows [Bowen and Ruelle 1975]:

$$
\varphi^{u}(\rho):=-\frac{d}{d \tau}\left(\operatorname{det}\left(\left.d_{\rho} g^{\tau}\right|_{E^{u}(\rho)}\right)\right)_{\tau=0} .
$$

3b. Kolmogorov-Sinai entropy. Let us recall a few facts about Kolmogorov-Sinai (or metric) entropy, which can be found for example in [Walters 1982]. Let $(X, \mathscr{B}, T, \mu)$ be a measurable dynamical system, and $\mathscr{P}:=\left(P_{\alpha}\right)_{\alpha \in I}$ a finite measurable partition of $X$, that is, a finite collection of measurable subsets that forms a partition. Each $P_{\alpha}$ is called an atom of the partition. With the convention $0 \log 0=0$, one defines

$$
H_{n}(\mu, T, \mathscr{P})=-\sum_{|\alpha|=n} \mu\left(P_{\alpha_{0}} \cap \cdots \cap T^{-(n-1)} P_{\alpha_{n-1}}\right) \log \mu\left(P_{\alpha_{0}} \cap \cdots \cap T^{-(n-1)} P_{\alpha_{n-1}}\right) .
$$

This quantity satisfies a subadditivity property

$$
H_{n+m}(\mu, T, \mathscr{P}) \leq H_{n}(\mu, T, \mathscr{P})+H_{m}\left(\mu, T, T^{-n} \mathscr{P}\right)=H_{n}(\mu, T, \mathscr{P})+H_{m}(\mu, T, \mathscr{P}) .
$$

The first inequality is true even if the probability measure $\mu$ is not $T$-invariant, while the last equality holds for $T$-invariant measures. A classical argument for subadditive sequences allows to define the 
quantity

$$
h_{\mathrm{KS}}(\mu, T, \mathscr{P}):=\lim _{n \rightarrow \infty} \frac{H_{n}(\mu, T, \mathscr{P})}{n},
$$

the Kolmogorov-Sinai entropy of $(T, \mu)$ with respect to the partition $\mathscr{P}$. The Kolmogorov-Sinai entropy $h_{\mathrm{KS}}(\mu, T)$ of $(\mu, T)$ is then defined as the supremum of $h_{\mathrm{KS}}(\mu, T, \mathscr{P})$ over all finite partitions $\mathscr{P}$ of $X$. In the case of a flow (for instance the dynamical system $\left(S^{*} M, g^{\tau}, \mu\right)$ ), we define the entropy $h_{\mathrm{KS}}\left(\mu,\left(g^{\tau}\right)\right):=h_{\mathrm{KS}}\left(\mu, g^{1}\right)$. Entropy can a priori be infinite. However, for a smooth flow on a compact finite dimensional manifold, entropy is bounded thanks to the Ruelle inequality [1978]. In the case of the geodesic flow on a negatively curved manifold, it reads

$$
h_{\mathrm{KS}}\left(\mu,\left(g^{\tau}\right)\right) \leq-\int_{S^{*} M} \varphi^{u}(\rho) d \mu(\rho),
$$

and equality holds if and only if $\mu$ is the disintegration $L$ of the Liouville measure on $S^{*} M$ (defined in Section 2a2) [Pesin 1977; Ledrappier and Young 1985].

Notation. In the rest of this paper, we will write $h_{\mathrm{KS}}(\mu)$ for $h_{\mathrm{KS}}\left(\mu,\left(g^{\tau}\right)\right)$, unless we want to consider a flow different from $\left(g^{\tau}\right)$.

3c. Topological pressure. To conclude this section, we introduce the topological pressure of the dynamical system $\left(S^{*} M, g^{\tau}\right)$ as the Legendre transform of the Kolmogorov-Sinai entropy [Walters 1982; Parry and Pollicott 1990; Pesin 1997]: for all $f \in \mathscr{C}^{0}\left(S^{*} M, \mathbb{R}\right)$,

$$
P(f)=P\left(S^{*} M,\left(g^{\tau}\right), f\right):=\sup \left\{h_{\mathrm{KS}}(\mu)+\int_{S^{*} M} f d \mu: \mu \in M\left(S^{*} M, g^{\tau}\right)\right\},
$$

where $\mathcal{M}\left(S^{*} M, g^{\tau}\right)$ is the set of probability measures on $S^{*} M$ invariant under the geodesic flow. This defines a continuous and convex function on $\mathscr{C}^{0}\left(S^{*} M, \mathbb{R}\right)$ [Walters 1982].

We shall be particularly interested in the behavior of $P(f)$ near $f=\varphi^{u}$. By the Ruelle inequality, we have $P\left(\varphi^{u}\right)=0$ (the sup defining $P\left(\varphi^{u}\right)$ is achieved at $\mu=L$; see Section 3b). Moreover, it can be proved that for any real-valued Hölder function $f$ on $S^{*} M$, the function $s \mapsto P\left(\varphi^{u}+s f\right)$ is real analytic on $\mathbb{R}$ [Bowen and Ruelle 1975; Ruelle 1976] and its derivatives of order 1 and 2 can be computed explicitly [Parry and Pollicott 1990].

We have $\left.\frac{d}{d s}\left(P\left(\varphi^{u}+s f\right)\right)\right|_{s=0}=\int_{S^{*} M} f d L$. If $\int_{S^{*} M} f d L=0$, the convex function $s \mapsto P\left(\varphi^{u}+s f\right)$ achieves its minimum at 0 . Moreover, if $\int_{S^{*} M} f d L=0$, then we have

$$
\left.\frac{d^{2}}{d s^{2}}\left(P\left(\varphi^{u}+s f\right)\right)\right|_{s=0}=\sigma^{2}(f),
$$

where

$$
\sigma^{2}(f):=\lim _{T \rightarrow+\infty} \frac{1}{T} \int_{S^{*} M}\left(\int_{0}^{T} f \circ g^{\tau}(\rho) d \tau\right)^{2} d L(\rho)
$$

is called the dynamical variance of the function $f$. It is known that $\sigma^{2}(f)$ vanishes if and only if $f$ is of the form $f=\left.\frac{d}{d \tau}\left(h \circ g^{\tau}\right)\right|_{\tau=0}$ for some function $h$. In this case, one says that $f$ is a coboundary. 
3d. Kifer's large deviation upper bound. We shall use the following result, due to Kifer [1992], and valid for more general Anosov flows:

$$
\lim _{T \rightarrow \infty} \frac{1}{T} \log \int_{S^{*} M} \exp \left(\int_{0}^{T} a \circ g^{\tau}(\rho) d \tau\right) d L(\rho)=P\left(a+\varphi^{u}\right),
$$

for all continuous $a$. In fact, we will only use that the lim sup is uniform for $a$ running over compact sets in the $\mathscr{C}^{1}$ topology (this property can be derived from the proof of [Kifer 1992, Theorem 3.2]).

Remark 7. This result implies the following strengthened version of the Birkhoff ergodic theorem. Fix $a$ such that $\int_{S^{*} M} a d L=0$, and fix $\delta>0$. Then

$$
\begin{aligned}
\limsup \frac{1}{T} \log L\left(\left\{\rho \in S^{*} M: \frac{1}{T} \int_{0}^{T} a \circ g^{\tau}(\rho) d \tau>\delta\right\}\right) & \leq \inf _{s \geq 0}\left\{-s \delta+P\left(s a+\varphi^{u}\right)\right\} \\
& =\inf _{s \in \mathbb{R}}\left\{-s \delta+P\left(s a+\varphi^{u}\right)\right\}=H(\delta) .
\end{aligned}
$$

Similarly, for $\delta<0$, one has

$$
\limsup \frac{1}{T} \log L\left(\left\{\rho \in S^{*} M: \frac{1}{T} \int_{0}^{T} a \circ g^{\tau}(\rho) d \tau<\delta\right\}\right) \leq H(\delta) .
$$

The function $-H$, which is the Legendre transform of $s \mapsto P\left(\varphi^{u}+s a\right)$, satisfies $H(\delta)=0$, is convex and is positive for $\delta \neq 0$ (it is infinite for $\delta \neq 0$ if $a$ is a coboundary).

\section{Examples of generalized orthonormal families}

In this section, we provide two examples of GOF and show how Theorem 2.2 applies to them. Our examples are of distinct types: basis of eigenvectors of $\Delta$ and truncated Dirac distributions. In the first example, Theorem 2.2 provides a strengthened version of Shnirelman's theorem for Anosov flows.

4a. Orthonormal basis of eigenvectors. Consider $\left(\psi_{n}\right)_{n \in \mathbb{N}}$, an orthonormal basis of $L^{2}(M)$ made of eigenfunctions of $\Delta$, that is, there exists a sequence $0=\lambda_{0}<\lambda_{1} \leq \cdots \leq \lambda_{n} \leq \cdots$ such that for every $n$ in $\mathbb{N}$,

$$
\Delta \psi_{n}=-\lambda_{n}^{2} \psi_{n}
$$

For $\hbar>0$, we take $\Omega_{\hbar}:=\left\{n \in \mathbb{N}: \hbar^{2} \lambda_{n}^{2} \in[1-\alpha \hbar, 1+\alpha \hbar]\right\}$, where $\alpha$ is some fixed positive number. In this case, the probability measure is given by $\mathbb{P}_{\hbar}:=\frac{1}{\left|\Omega_{\hbar}\right|} \sum_{n \in \Omega_{\hbar}} \delta_{n}$ and the measurable map is given by $u_{\hbar}(n):=\psi_{n}$. Applying Corollary 2.3 to this example, we find that for every $a$ in $\mathscr{C}_{o}^{\infty}\left(T^{*} M\right)$, and for every $\delta>0$, there exists $\tilde{H}(\delta)>0$ such that

$$
\frac{1}{\left|\Omega_{\hbar}\right|}\left|\left\{n \in \Omega_{\hbar}:\left|\mu_{\hbar, n}(a)-\int_{S^{*} M} a d L\right| \geq \delta\right\}\right|=\sigma_{a, \delta}\left(\hbar^{\tilde{H}(\delta)}\right) .
$$

Shnirelman's theorem provides a $o_{a, \delta}(1)$, and using the results from [Zelditch 1994] on eigenfunctions of $\Delta$, one can obtain a $O_{a, \delta, p}\left(|\log \hbar|^{-p}\right)$ for arbitrarily large $p$. 
4b. Truncated Dirac distributions. The second class of examples we will consider is given by families of vectors constructed from the Dirac distributions. For $y$ in $M$, we denote by $\delta_{y}$ the Dirac distribution given by $\left\langle\delta_{y}, f\right\rangle:=f(y)$ (where $f$ is in $\mathscr{C}^{\infty}(M)$ ). To construct our GOF, we will project $\delta_{y}$ on $L^{2}(M)$. To do this, recall that we have defined $I(\hbar):=[a(\hbar), b(\hbar)]$, where $b(\hbar)-a(\hbar) \geq 2 \alpha \hbar$, and that we have defined $N(I(\hbar)):=\left|\left\{n: \hbar^{2} \lambda_{n}^{2} \in I(\hbar)\right\}\right|$. Using this notation, we can introduce a truncated Dirac distribution by

$$
\delta_{y}^{\hbar}:=\left(\frac{\operatorname{Vol}_{M}(M)}{N(I(\hbar))}\right)^{1 / 2} \mathbb{1}_{I(\hbar)}\left(-\hbar^{2} \Delta\right) \delta_{y} .
$$

According to (global and local) Weyl laws from [Duistermaat and Guillemin 1975] and from [Sogge and Zelditch 2002, Theorem 1.2]), we know that in the Anosov case,

$$
\left(M, \frac{\operatorname{Vol}_{M}}{\operatorname{Vol}_{M}(M)}, \delta_{y}^{\hbar}\right) \text { is a GOF in the spectral window } I(\hbar) .
$$

Applying Corollary 2.3 to this example, we find that for every $a$ in $\mathscr{C}_{o}^{\infty}\left(T^{*} M, \mathbb{C}\right)$, for every $\theta$ in $L^{1}\left(\mathbb{R}, \mathbb{R}_{+}\right)$and for every $\delta>0$, there exists $\tilde{H}(\delta)>0$ such that

$$
\operatorname{Vol}_{M}\left(\left\{y \in M:\left|\mu_{\hbar, y}(\theta \otimes a)-\int_{S^{*} M} a d L \int_{\mathbb{R}} \theta(t) d t\right| \geq \delta\right\}\right):=\bigcirc_{a, \theta, \delta}\left(\hbar^{\tilde{H}(\delta)}\right) .
$$

Thus, if we choose $y$ randomly on $M$ according to the volume measure, and consider the solution of the Schrödinger equation $e^{l t \Delta / 2} \delta_{y}^{\hbar}$, our result says that we have convergence of the associated semiclassical measure to the uniform measure, for most $y$ (in the probability sense, and with an explicit bound) as $\hbar$ tends to 0 . Taking a subsequence $\left(\hbar_{n}\right)_{n}$ that tends to 0 fast enough, we can apply the Borel-Cantelli lemma and derive convergence for almost every $y$ [Rivière 2009]. An interesting question would be to understand more precisely for which subsequences $\left(\hbar_{n}\right)$ we have convergence for almost every $y$.

4c. Coherent states. Similar results could, in principle, apply to bases of coherent states (e.g., gaussian states). Such bases can be constructed easily in euclidean situations; see [Rivière 2009] for an application of Theorem 2.2 to the "cat map" toy model. However, on an arbitrary manifold, it seems difficult to construct bases of coherent states meeting all the requirements of the definition of a GOF, which are actually quite strong.

\section{Proof of Theorem 2.2}

The proof has two steps. To begin with, we combine the Bienaymé-Chebyshev inequality and the Egorov theorem to obtain a first bound (Section 5b). Then we apply a large deviations estimate due to Kifer [1992] to obtain a bound in terms of the topological pressure. This proof follows the steps of Zelditch [1994], the new input being

- the use of the exponential function $x \mapsto e^{x}$ in Section $5 \mathrm{~b}$ instead of the power functions $x \mapsto x^{p}$;

- the use of Kifer's large deviation result for the geodesic flow instead of the central-limit theorem; ${ }^{1}$

\footnotetext{
${ }^{1}$ Rigorously speaking, one cannot say that the LDP is stronger than the CLT. When the large deviation principle holds with a rate function that is $C^{2}$ and strictly convex, one usually expects to have a central limit theorem; the variance of the limiting
} 
- a more careful treatment of the trace asymptotics (Lemma 5.3) to make sure that the remainder term is not larger than the leading term for the symbols we consider.

We fix $\theta$ an element of $L^{1}\left(\mathbb{R}, \mathbb{R}_{+}\right)$such that $\int \theta(t) d t=1$. Let $a$ be an element in $\mathscr{C}_{o}^{\infty}\left(T^{*} M, \mathbb{R}\right)$ that satisfies $\int_{S^{*} M} a d L=0$. Recall that we defined

$$
\chi_{\max }:=\lim _{\tau \rightarrow \pm \infty} \frac{1}{\tau} \log \sup _{\rho \in S^{*} M}\left\|d_{\rho} g^{\tau}\right\| .
$$

Since the states $u_{\hbar}(\omega)$ are uniformly microlocalized in a thin neighborhood of $S^{*} M$, we can assume that $a$ is compactly supported in a tubular neighborhood $p^{-1}\left(\left[\frac{1}{2}-\eta, \frac{1}{2}+\eta\right]\right.$ ) of $S^{*} M$ (with $\eta>0$ arbitrarily small). Letting $\chi_{\eta}=\chi_{\max } \sqrt{1+2 \eta}$, we have, for all $\tau \in \mathbb{R}$, for all $\rho \in T^{*} M$ and for all $\alpha$,

$$
\left\|\partial^{\alpha}\left(a \circ g^{\tau}\right)(\rho)\right\| \leq C_{a, \alpha} e^{\chi_{\eta}|\alpha||\tau|} .
$$

5a. Long-time Egorov theorem. We fix $c$ such that $c \chi_{\eta}<\frac{1}{2}$. The positive quantization $\mathrm{Op}_{\hbar}^{+}$procedure described in Appendix A satisfies the following "long time Egorov property":

$$
\left\|U^{-\tau \hbar} \mathrm{Op}_{\hbar}^{+}(a) U^{\tau \hbar}-\mathrm{Op}_{\hbar}^{+}\left(a \circ g^{\tau}\right)\right\|_{L^{2}(M) \rightarrow L^{2}(M)}=O_{a}\left(\hbar^{1 / 2-v}\right) \quad \text { for all }|\tau| \leq c|\log \hbar|
$$

where $v:=c \chi_{\eta}$; see [Anantharaman and Nonnenmacher 2007].

Lemma 5.1. For every $\delta_{0}>0$, there exists $\hbar_{0}$ (depending on a, $\theta$ and $\delta_{0}$ ) such that for every $\hbar<\hbar_{0}$,

$$
\left\|\int \theta(t) U^{-t}\left(\mathrm{Op}_{\hbar}^{+}(a)-\frac{1}{2 T} \int_{-T}^{T} \mathrm{Op}_{\hbar}^{+}\left(a \circ g^{\tau}\right) d \tau\right) U^{t} d t\right\|_{L^{2}(M) \rightarrow L^{2}(M)} \leq \delta_{0} \quad \text { for every }|T| \leq c|\log \hbar| .
$$

Proof. The proof of this lemma relies on the application of the Egorov property (13). For $T$ a real number such that $|T| \leq c|\log \hbar|$, we have

$$
\int \theta(t) U^{-t}\left(\frac{1}{2 T} \int_{-T}^{T} \mathrm{Op}_{\hbar}^{+}\left(a \circ g^{\tau}\right) d \tau\right) U^{t} d t=\frac{1}{2 T} \int_{-T}^{T} \int \theta(t) U^{-t-\tau \hbar} \mathrm{Op}_{\hbar}^{+}(a) U^{t+\tau \hbar} d t d \tau+\mathrm{O}_{a}\left(\hbar^{1 / 2-v}\right) .
$$

We make the change of variables $t^{\prime}=t+\tau \hbar$ and use the fact that $\left.\| \theta(\cdot)-\theta(\cdot-\tau)\right) \|_{L^{1}} \underset{\tau \rightarrow 0}{\longrightarrow} 0$ to conclude.

5b. Bienaymé-Chebyshev and Jensen's inequality. For simplicity of notation, we will denote the quantity we want to bound as follows:

$$
\mathbb{P}_{\hbar}(\theta \otimes a, \delta):=\mathbb{P}_{\hbar}\left(\left\{\omega \in \Omega_{\hbar}: \mu_{\hbar, \omega}(\theta \otimes a) \geq \delta\right\}\right)
$$

The first step is to combine the previous lemma with the Bienaymé-Chebyshev inequality to obtain a bound on $\mathbb{P}_{\hbar}(\theta \otimes a, \delta)$.

gaussian being the second derivative of the rate function at its minimum. Formally, one makes a Taylor expansion of order 2 of the LDP near the minimum of the rate function to derive a gaussian behavior. However, the implementation of this idea requires a very precise and strong version of the LDP, and in practice one prefers to prove the CLT independently. 
Lemma 5.2. Let $\delta, \delta_{0}>0$ be arbitrary positive numbers. For $s \in \mathbb{R}$, let

$$
a_{s}(T(\hbar), \rho):=\exp \left(s \int_{-T(\hbar)}^{T(\hbar)} a \circ g^{\tau}(\rho) d \tau\right)
$$

where $T(\hbar)=c|\log \hbar|\left(\right.$ and $c$ is such that $\left.c \chi_{\eta}<1 / 2\right)$. Then, given $s>0$ and for $\hbar$ small enough, one has

$$
\mathbb{P}_{\hbar}(\theta \otimes a, \delta) \leq 2 \frac{e^{\left(-2 \delta+4 \delta_{0}\right) s T(\hbar)}}{N(I(\hbar))} \operatorname{Tr}\left[\mathbb{1}_{I(\hbar)}\left(-\hbar^{2} \Delta\right) \mathrm{Op}_{\hbar}^{+}\left(a_{s}(T(\hbar), \cdot)\right)\right]
$$

Proof. Let $s>0$. A direct application of the Bienaymé-Chebyshev inequality allows us to write

$$
\mathbb{P}_{\hbar}(\theta \otimes a, \delta):=\mathbb{P}_{\hbar}\left(\left\{\omega \in \Omega_{\hbar}: \mu_{\hbar, \omega}(\theta \otimes a) \geq \delta\right\}\right) \leq e^{-2 s \delta T(\hbar)} \int_{\Omega_{\hbar}} \exp \left(2 s T(\hbar) \mu_{\hbar, \omega}(\theta \otimes a)\right) d \mathbb{P}_{\hbar}(\omega) .
$$

We can now use Lemma 5.1 and deduce that, for $\hbar$ small enough,

$$
\mathbb{P}_{\hbar}(\theta \otimes a, \delta) \leq e^{-2 s \delta T(\hbar)} \int_{\Omega_{\hbar}} \exp \left(s \mu_{\hbar, \omega}\left(\theta \otimes\left(\int_{-T(\hbar)}^{T(\hbar)} a \circ g^{\tau} d \tau\right)\right)+2 s \delta_{0} T(\hbar)\left\|u_{\hbar}(\omega)\right\|^{2}\right) d \mathbb{P}_{\hbar}(\omega)
$$

Using the fact that $\left\|u_{\hbar}(\omega)\right\|=1+o(1)$ uniformly for $\omega$ in $\Omega_{\hbar}$, the quantity $e^{2 s \delta_{0} T(\hbar)\left\|u_{\hbar}(\omega)\right\|^{2}}$ is uniformly bounded by $e^{3 s \delta_{0} T(\hbar)}$ for $\hbar$ small enough. The map $x \mapsto e^{s x}$ is convex and we can use Jensen's inequality to write

$$
\mathbb{P}_{\hbar}(\theta \otimes a, \delta) \leq e^{s\left(-2 \delta+3 \delta_{0}\right) T(\hbar)} \int_{\Omega_{\hbar}} \mu_{\hbar, \omega}\left(\exp \left(s \mu_{\hbar, \omega}(\theta \otimes 1)\left(\int_{-T(\hbar)}^{T(\hbar)} a \circ g^{\tau} d \tau\right)\right) \otimes \theta\right) \frac{d \mathbb{P}_{\hbar}(\omega)}{\mu_{\hbar, \omega}(\theta \otimes 1)}
$$

Using again that $\left\|u_{\hbar}(\omega)\right\|=1+o(1)$ uniformly for $\omega$ in $\Omega_{\hbar}$ and that $\theta$ is nonnegative and $\int \theta(t) d t=1$, one has

$$
\mu_{\hbar, \omega}(\theta \otimes 1)=1+o(1)
$$

uniformly in $\omega$ for $\hbar$ small enough. All this can be summarized as follows:

$$
\mathbb{P}_{\hbar}(\theta \otimes a, \delta) \leq 2 e^{s\left(-2 \delta+4 \delta_{0}\right) T(\hbar)} \int_{\Omega_{\hbar}} \mu_{\hbar, \omega}\left(\theta \otimes a_{s}(T(\hbar), \cdot)\right) d \mathbb{P}_{\hbar}(\omega)
$$

Note that the function $a_{S}(T(\hbar), \cdot)$ belongs to the class of symbols $S_{v}^{0, k_{0}}\left(T^{*} M\right)$, where $v:=c \chi_{\eta}<1 / 2$ and $k_{0}:=2 c s\|a\|_{\infty}$ (Appendix A); moreover $a_{s}(T(\hbar), \cdot)$ is constant in a neighborhood of infinity. The previous inequality can be rewritten as

$$
\mathbb{P}_{\hbar}(\theta \otimes a, \delta) \leq 2 e^{\left(-2 \delta+4 \delta_{0}\right) s T(\hbar)} \int \theta(t) \int_{\Omega_{\hbar}}\left\langle u_{\hbar}(\omega)\left|U^{-t} \mathrm{Op}_{\hbar}^{+}\left(a_{s}(T(\hbar), \cdot)\right) U^{t}\right| u_{\hbar}(\omega)\right\rangle d \mathbb{P}_{\hbar}(\omega) d t
$$

We recall that if $\left(u_{\hbar}(\omega)\right)_{\omega \in\left(\Omega_{\hbar}, \mathbb{P}_{h}\right)}$ is a GOF then for every $t$ in $\mathbb{R}, \quad\left(U^{t} u_{\hbar}(\omega)\right)_{\omega \in\left(\Omega_{h}, \mathbb{P}_{h}\right)}$ is also a GOF Using point 3 of the definition of a GOF, we get for $\hbar$ small enough the bound

$$
\mathbb{P}_{\hbar}(\theta \otimes a, \delta) \leq \frac{2 e^{\left(-2 \delta+4 \delta_{0}\right) s T(\hbar)}}{N(I(\hbar))} \operatorname{Tr}\left[\mathbb{1}_{I(\hbar)}\left(-\hbar^{2} \Delta\right) \mathrm{Op}_{\hbar}^{+}\left(a_{S}(T(\hbar), \cdot)\right)\right]
$$


5c. Trace asymptotics. We now have to estimate (from above) the trace

$$
\operatorname{Tr}\left[\mathbb{1}_{I(\hbar)}\left(-\hbar^{2} \Delta\right) \mathrm{Op}_{\hbar}^{+}\left(a_{s}(T(\hbar), \cdot)\right)\right]
$$

We first underline that, for every $\hbar>0$, there exist energy levels $E_{1}<\cdots<E_{P}$ (depending on $\hbar$ ) such that

$$
I(\hbar)=[a(\hbar), b(\hbar)] \subset \bigsqcup_{p=1}^{P}\left[E_{p}-\alpha \hbar, E_{p}+\alpha \hbar\right) \subset[a(\hbar)-\alpha \hbar, b(\hbar)+\alpha \hbar],
$$

for some fixed positive $\alpha$. Note that $P=\mathcal{O}((b(\hbar)-a(\hbar)) / \hbar)$. We decompose (15) into

$$
\sum_{p=1}^{P} \operatorname{Tr}\left[\mathbb{1}_{\left[E_{p}-\alpha \hbar, E_{p}+\alpha \hbar\right)}\left(-\hbar^{2} \Delta\right) \mathrm{Op}_{\hbar}^{+}\left(a_{s}(T(\hbar), \cdot)\right)\right] .
$$

We shall bound each term of the previous sum (uniformly with respect to $p$ ), using standard trace estimates, and then sum over $p$. We consider for instance the interval $[1-\alpha \hbar, 1+\alpha \hbar)$, and recall how to determine the asymptotic behavior of

$$
\operatorname{Tr}\left[\mathbb{1}_{[1-\alpha \hbar, 1+\alpha \hbar)}\left(-\hbar^{2} \Delta\right) \mathrm{Op}_{\hbar}^{+}\left(a_{S}(T(\hbar), \cdot)\right)\right]
$$

Introduce a function $f$ that is $\mathscr{C}^{\infty}$, compactly supported in a small neighborhood of 1 , equal to 1 in a neighborhood of 1 and taking values in $[0,1]$. We shall also use a function $\chi$ in $\mathscr{S}\left(\mathbb{R}^{d}\right)$ whose Fourier transform is compactly supported in a small neighborhood of 0 , containing no period of the closed geodesics of $\left(g^{\tau}\right)$ on $S^{*} M$. We assume that $\chi \geq 0$ and that it is greater than 1 on $[-\alpha, \alpha]$. Using the fact that the quantization is positive, we can bound the previous quantity as

$$
\operatorname{Tr}\left[\mathbb{1}_{[1-\alpha \hbar, 1+\alpha \hbar)}\left(-\hbar^{2} \Delta\right) \mathrm{Op}_{\hbar}^{+}\left(a_{s}(T(\hbar), \cdot)\right)\right] \leq \operatorname{Tr}\left[f\left(-\hbar^{2} \Delta\right) \chi\left(\frac{-\hbar^{2} \Delta-1}{\hbar}\right) \mathrm{Op}_{\hbar}^{+}\left(a_{s}(T(\hbar), \cdot)\right)\right]
$$

The study of this last quantity now follows well-known lines. We use the Fourier inversion formula,

$$
2 \pi \chi\left(\frac{E-1}{\hbar}\right)=\int_{\mathbb{R}} e^{l(E-1) \tau / \hbar} \hat{\chi}(\tau) d \tau .
$$

As a consequence, the right hand side of (16) can be written as

$$
\frac{1}{2 \pi} \int_{\mathbb{R}} e^{-l \tau / \hbar} \operatorname{Tr}\left(\mathrm{Op}_{\hbar}^{+}\left(a_{S}(T(\hbar), \cdot)\right) U^{2 \tau \hbar} f\left(-\hbar^{2} \Delta\right)\right) \hat{\chi}(\tau) d \tau .
$$

The asymptotic behavior of the trace comes from an asymptotic expansion of the kernel of the operator $\mathrm{Op}_{\hbar}^{+}\left(a_{s}(T(\hbar), \cdot)\right) U^{2 \tau \hbar} f\left(-\hbar^{2} \Delta\right)$. This expansion is given by the theory of Fourier integral operators [Dimassi and Sjöstrand 1999, Chapter 11; Zworski 2012, Chapter 10]. The trace is then expressed as the integral of the kernel over the diagonal, and the asymptotic behavior of this integral is determined by the method of stationary phase [Dimassi and Sjöstrand 1999, Chapter11]. 
Lemma 5.3. For every integer $N \geq 1$, we have

$$
\begin{gathered}
\operatorname{Tr}\left[f\left(-\hbar^{2} \Delta\right) \chi\left(\frac{-\hbar^{2} \Delta-1}{\hbar}\right) \operatorname{Op}_{\hbar}^{+}\left(a_{s}(T(\hbar), \cdot)\right)\right] \\
=\frac{1}{(2 \pi \hbar)^{d-1}}\left(\sum_{n=0}^{N-1} \hbar^{n} \int_{S^{*} M} D^{2 n} a_{s}(T(\hbar), \rho) d L(\rho)+O_{a, \chi, \theta, N}\left(\hbar^{N(1-2 v)-\beta \nu-k_{0}}\right)\right)
\end{gathered}
$$

where $\beta>0$ depends only on the dimension of $M$, and where $D^{2 n}$ is a differential operator of order $2 n$ on $T^{*} M$ (depending on the cutoff functions and on the choice of the quantization $\mathrm{Op}_{\hbar}^{+}$).

There are many references for these kind of estimates. For instance, a very similar calculation is done by Schubert [2006, Proposition 1] (he stops at $N=1$ but the stationary phase method actually provides asymptotic expansions at any order).

Recall that $v=c \chi_{\eta}<\frac{1}{2}$. It is important here to note that $a_{s}(T(\hbar), \cdot)$ belongs to the class $S_{v}^{0, k_{0}}\left(T^{*} M\right)$, and that the observable $a_{s}(T(\hbar), x, \xi)$ satisfies the particular property that $D^{2 n} a_{s}(T(\hbar), \rho)$ is of the form $a_{s}(T(\hbar), x, \xi) b_{2 n}(x, \xi)$, with $\left\|b_{2 n}\right\|_{\infty}=\mathcal{O}\left(|s|^{2 n} \hbar^{-2 n v}\right)$ as $\hbar \rightarrow 0$ and $s \rightarrow \infty$. If $s$ stays in a bounded interval, and if we choose $N$ large enough accordingly, this implies that

$\operatorname{Tr}\left[f\left(-\hbar^{2} \Delta\right) \chi\left(\frac{-\hbar^{2} \Delta-1}{\hbar}\right) \mathrm{Op}_{\hbar}^{+}\left(a_{s}(T(\hbar), \cdot)\right)\right] \leq \frac{1}{(2 \pi \hbar)^{d-1}}\left(\int_{S^{*} M} a_{S}(T(\hbar), \rho) d L(\rho)\right)\left(1+\mathcal{O}\left(\hbar^{1-2 v}\right)\right)$.

Combing this with Lemma 5.2 and using the Weyl law (4), we finally have, for every $N \geq 1$ and $\hbar$ small enough,

$$
\mathbb{P}_{\hbar}(\theta \otimes a, \delta) \leq C e^{\left(-2 \delta+4 \delta_{0}\right) s T(\hbar)}\left(\int_{S^{*} M} a_{s}(T(\hbar), \rho) d L(\rho)\right)\left(1+\mathcal{O}\left(\hbar^{1-2 v}\right)\right),
$$

for some constant $C$ that does not depend on $\hbar$.

5d. A large deviations bound. To conclude, we use Kifer's large deviations result (12). For our proof, we only need an upper bound on the quantity

$$
\int_{S^{*} M} \exp \left(s \int_{-T}^{T} a \circ g^{\tau}(\rho) d \tau\right) d L(\rho)
$$

Compared with (12), there is a parameter $s$ in the exponential that stays in a bounded interval $I$. Following the proof of the upper bound (12) in [Kifer 1992, Section 3], one can say that for every $\delta^{\prime}>0$ and any bounded interval $I$ in $\mathbb{R}_{+}$, there exists $c_{\delta^{\prime}}>0$ and $n\left(\delta^{\prime}, I\right) \in \mathbb{N}$ such that for every $T \geq n\left(\delta^{\prime}, I\right)$ and every $s$ in $I$,

$$
\int_{S^{*} M} \exp \left(s \int_{-T}^{T} a \circ g^{\tau}(\rho) d \tau\right) d L(\rho) \leq c_{\delta^{\prime}} e^{T \delta^{\prime}} e^{2 T P\left(s a+\varphi^{u}\right)} .
$$

This last bound will allow us to conclude. In fact, combining this inequality to the bound (17) on $\mathbb{P}_{\hbar}(\theta \otimes a, \delta)$, we find that

$$
\mathbb{P}_{\hbar}(\theta \otimes a, \delta) \leq C e^{\left(-2 \delta+4 \delta_{0}\right) s T(\hbar)} e^{T(\hbar) \delta^{\prime}} e^{2 T(\hbar) P\left(s a+\varphi^{u}\right)},
$$


where the constant $C$ depends on the various parameters but not on $\hbar$. This implies

$$
\limsup _{\hbar \rightarrow 0} \frac{\log \left(\mathbb{P}_{\hbar}(\theta \otimes a, \delta)\right)}{c|\log \hbar|} \leq \delta^{\prime}+\left(-2 \delta+4 \delta_{0}\right) s+2 P\left(s a+\varphi^{u}\right) .
$$

This last inequality holds for any $\delta_{0}>0$ and any $\delta^{\prime}>0$. It implies that for every $s>0$ in the interval $I$,

$$
\limsup _{\hbar \rightarrow 0} \frac{\log \left(\mathbb{P}_{\hbar}(\theta \otimes a, \delta)\right)}{c|\log \hbar|} \leq-2 s \delta+2 P\left(s a+\varphi^{u}\right) \quad \text { for all } c \in\left(0, \frac{1}{2 \chi_{\max }}\right) .
$$

In particular, we find that

$$
\limsup _{\hbar \rightarrow 0} \frac{\log \left(\mathbb{P}_{\hbar}(\theta \otimes a, \delta)\right)}{|\log \hbar| /\left(2 \chi_{\max }\right)} \leq 2 \inf _{s \in \mathbb{R}_{+}}\left\{-s \delta+P\left(s a+\varphi^{u}\right)\right\} \quad \text { for all } \delta \in \mathbb{R} .
$$

Since $\delta>0$, we have $\inf _{s \in \mathbb{R}_{+}}\left\{-s \delta+P\left(s a+\varphi^{u}\right)\right\}=\inf _{s \in \mathbb{R}}\left\{-s \delta+P\left(s a+\varphi^{u}\right)\right\}$. This concludes the proof of Theorem 2.2.

\section{Proof of Theorem 2.4}

In this section, we assume that $M$ has constant sectional curvature -1 , and we fix two energy levels $0 \leq E_{1}<E_{2}$ and consider a sequence $\left(u_{\hbar}\right)_{\hbar \rightarrow 0^{+}}$in $L^{2}(M)$ that satisfies

$$
\lim _{\hbar \rightarrow 0}\left\|\left(\operatorname{Id}_{L^{2}(M)}-\mathbb{1}_{\left[E_{1}, E_{2}\right]}\left(-\hbar^{2} \Delta\right)\right) u_{\hbar}\right\|_{L^{2}(M)}=0 .
$$

Moreover, we suppose that $\left\|u_{\hbar}\right\|_{L^{2}(M)}=1$. The proof follows essentially the same lines as the one in [Anantharaman and Nonnenmacher 2007], and we refer the reader to that paper for a detailed account.

6a. Quantum partitions. As usual when computing the Kolmogorov-Sinai entropy, we start by decomposing the manifold $M$ into finitely many pieces (of small diameter). Let $\left(P_{k}\right)_{k=1, \ldots, K}$ be a family of smooth real functions on $M$ such that

$$
\sum_{k=1}^{K} P_{k}^{2}(x)=1 \text { for all } x \in M .
$$

Later on we will assume that the diameters of the supports of the $P_{k}$ are small enough. We shall denote by $\hat{P}_{k}$ the operator of multiplication by $P_{k}(x)$ on the Hilbert space $L^{2}(M)$. We denote the Schrödinger flow by $U^{t}=\exp (\imath t \Delta / 2)$. With no loss of generality, we will assume that the injectivity radius of $M$ is greater than 2, and work with this propagator at time $\hbar$, that is, $U^{\hbar}$. This unitary operator is a Fourier integral operator associated with the geodesic flow $g^{1}$ taken at time $\tau=1$. As one does to compute the Kolmogorov-Sinai entropy of an invariant measure, we define a new quantum partition of unity by evolving and refining the initial partition under the quantum evolution. For each time $n \in \mathbb{N}$ and any sequence of symbols $\alpha=\left(\alpha_{0}, \ldots, \alpha_{n-1}\right)$, where $\alpha_{i} \in[1, K]$ (we say that the sequence $\alpha$ is of length $|\alpha|=n)$, we define the operators

$$
\pi_{\alpha}=\hat{P}_{\alpha_{n-1}}(n-1) \hat{P}_{\alpha_{n-2}}(n-2) \cdots \hat{P}_{\alpha_{0}} .
$$


Throughout the paper we use the notation $\hat{A}(\tau)=U^{-\tau \hbar} \hat{A} U^{\tau \hbar}$ for the quantum evolution of an operator $\hat{A}$. From (19) and the unitarity of $U^{t}$, the family of operators $\left\{\pi_{\alpha}:|\alpha|=n\right\}$ obviously satisfies the resolution of identity $\sum_{|\alpha|=n} \pi_{\alpha} \pi_{\alpha}^{*}=\operatorname{Id}_{L^{2}(M)}$. We also have $\sum_{|\alpha|=n} \pi_{\alpha}^{*} \pi_{\alpha}=\operatorname{Id}_{L^{2}(M)}$.

6b. Quantum entropy, and entropic uncertainty principle. For each time $n$ and each normalized $\phi$ in $L^{2}(M)$, we define two quantities that are noncommutative analogues of the entropy (9):

$$
\begin{aligned}
& h_{n}^{-}(\phi)=-\sum_{|\alpha|=n}\left\|\pi_{\alpha}^{*} \phi\right\|^{2} \log \left(\left\|\pi_{\alpha}^{*} \phi\right\|^{2}\right), \\
& h_{n}^{+}(\phi)=-\sum_{|\alpha|=n}\left\|\pi_{\alpha} \phi\right\|^{2} \log \left(\left\|\pi_{\alpha} \phi\right\|^{2}\right) .
\end{aligned}
$$

In all that follows, the integer $n$ is of order $\tilde{c}|\log \hbar|$ (with $\tilde{c}>0$ to be chosen later), and thus the number of terms in the sum $\sum_{|\alpha|=n}$ is of order $\hbar^{-K_{0}}$ for some $K_{0}>0$. The following is proved in [Anantharaman and Nonnenmacher 2007], using the entropic uncertainty principle of [Maassen and Uffink 1988].

Proposition 6.1. Let $\chi$ be real-valued, smooth, compactly supported function on $\mathbb{R}$. Define

$$
c(\chi, n):=\max _{|\alpha|=\left|\alpha^{\prime}\right|=n}\left(\left\|\pi_{\alpha^{\prime}}(n) \pi_{\alpha} \chi\left(-\hbar^{2} \Delta\right)\right\|\right) .
$$

Then for any $\hbar>0$ and $L>0$, and for any normalized state $\phi$ satisfying

$$
\sup _{|\alpha|=n}\left\|\left(I-\chi\left(-\hbar^{2} \Delta\right)\right) \pi_{\alpha}^{*} \phi\right\| \leq \hbar^{L}
$$

we have

$$
h_{n}^{+}\left(U^{n \hbar} \phi\right)+h_{n}^{-}(\phi) \geq-2 \log \left(c(\chi, n)+h^{L-K_{0}}\right) .
$$

Finally everything boils down to the main estimate:

Theorem 6.2 [Anantharaman 2008; 2011; Anantharaman and Nonnenmacher 2007]. If the diameters of the supports of the functions $P_{k}$ are small enough (compared with the injectivity radius), the following holds.

For $E>0$ and $0<\varepsilon<E$, choose $\chi$ smooth, compactly supported in $[E-\varepsilon, E+\varepsilon]$, and such that $\|\chi\|_{\infty} \leq 1$. For any $\tilde{c}>0$, there exists $\hbar_{\tilde{c}}>0$ such that, for all $\hbar<\hbar_{\tilde{c}}$, for $n \leq \tilde{c}|\log \hbar|$, and for any pair $\alpha, \alpha^{\prime}$ of sequences of length $n$,

$$
\left\|\pi_{\alpha^{\prime}}(n) \pi_{\alpha} \chi\left(-\hbar^{2} \Delta\right)\right\| \leq C \hbar^{-(d-1) / 2} e^{-n(d-1) \sqrt{E-\varepsilon}} .
$$

(The constant $C$ is an absolute constant).

Remark 8. This result is an improvement of the estimate of [Anantharaman 2008] (where the prefactor was only $\hbar^{-d / 2}$ ) and [Anantharaman and Nonnenmacher 2007] (where the support of $\chi$ was assumed to shrink with $\hbar$ ). Proving Theorem 2.4 using the weaker results of these papers turned out to be more painful than reproving Theorem 6.2 directly. This proof is provided in [Anantharaman 2011, Section 5]. Unfortunately, the arguments of there are specific to constant curvature, although we believe the result should also hold in variable negative curvature (parts of the proof rely on the fact that the stable and 
unstable foliations of the geodesic flow are smooth). Thus, if we wanted to extend Theorem 2.4 so as to get the results claimed in Remark 2, we would have to use the hyperbolic dispersive estimate in the form used in [Anantharaman and Nonnenmacher 2007], which would need a rather different, and more technical, presentation.

In what follows, the integer $n$ will always be taken equal to $\lfloor\tilde{c}|\log \hbar|\rfloor$, where $\tilde{c}$ will be fixed in the next section. We assume that $L$ is large enough so that $\hbar^{L-K_{0}}$ is negligible in comparison with $\hbar^{-(d-1) / 2} e^{-n(d-1) \sqrt{E-\varepsilon}}$. As a corollary of Theorem 6.2 and Proposition 6.1, we have this:

Corollary 6.3. Let $\left(\phi_{\hbar}\right)_{\hbar \rightarrow 0}$ be a sequence of normalized states satisfying the assumptions of 6.1, with $L$ large enough that $\hbar^{L-K_{0}}$ is negligible in comparison with $\hbar^{-(d-1) / 2} e^{-n(d-1) \sqrt{E-\varepsilon}}$ for $n=\lfloor\tilde{c}|\log \hbar|\rfloor$. Then, in the semiclassical limit, the entropies of $\phi_{\hbar}$ at time $n=\lfloor\tilde{c}|\log \hbar|\rfloor$ satisfy

$$
\frac{h_{n}^{+}\left(U^{n \hbar} \phi_{\hbar}\right)+h_{n}^{-}\left(\phi_{\hbar}\right)}{2 n} \geq(d-1) \sqrt{E-\varepsilon}-\frac{(d-1)}{2 \tilde{c}}+\mathcal{O}\left(n^{-1}\right) .
$$

6c. Subadditivity until the Ehrenfest time. In this section, we fix a sequence of normalized states $\left(\phi_{\hbar}\right)_{\hbar \rightarrow 0}$ satisfying (24) ( $\chi$ is always assumed to be supported in $[E-\varepsilon, E+\varepsilon]$ ). We fix some arbitrary $\delta>0$, and introduce the Ehrenfest time,

$$
n_{\mathrm{Ehr}}(\hbar, E, \varepsilon):=\left\lfloor\frac{(1-\delta)|\log \hbar|}{\sqrt{E+\varepsilon}}\right\rfloor .
$$

Remark 9. The Ehrenfest time is the largest time on which the (noncommutative) dynamical system formed by the flow $\left(U^{\tau \hbar}\right)$ acting on pseudodifferential operators (supported in $\left\{\|\xi\|^{2} \in[E-\varepsilon, E+\varepsilon]\right\}$ ) is commutative, up to small errors going to 0 with $\hbar$.

We take $n=n_{\mathrm{Ehr}}(\hbar, E, \varepsilon)$ (in other words, we take $\tilde{c}=(1-\delta) / \sqrt{E+\varepsilon}$ ), and we use a subadditivity property of the entropies $h_{n}^{+}$and $h_{n}^{-}$to go from (26) for $n=n_{\mathrm{Ehr}}(\hbar, E, \varepsilon)$ to a fixed, arbitrary, integer $n_{0}$. The proof of the next proposition is given in [Anantharaman and Nonnenmacher 2007] in the case when $\phi_{\hbar}$ is an eigenfunction of $\Delta$. It can easily be adapted to the case of an arbitrary $\phi_{\hbar}$ and yields this:

Proposition 6.4 (subadditivity). Let $E \geq 0$ and $\varepsilon>0$. For $\delta>0$ arbitrary, define the Ehrenfest time $n_{\mathrm{Ehr}}(\hbar, E, \varepsilon)$ as in (27). Let $\left(\phi_{\hbar}\right)_{\hbar \rightarrow 0}$ be a normalized family satisfying (24), where $\chi$ is supported in $[E-\varepsilon, E+\varepsilon]$, and $L$ is chosen large enough.

For any $n_{0} \in \mathbb{N}$, there exists a positive $R_{n_{0}}(\hbar)$, with $R_{n_{0}}(\hbar) \rightarrow 0$ as $\hbar \rightarrow 0$, such that for any $\hbar \in(0,1]$ and any $n_{0}, m \in \mathbb{N}$ with $n_{0}+m \leq n_{\mathrm{Ehr}}(\hbar)$, we have

$$
\begin{aligned}
& h_{n_{0}+m}^{+}\left(\phi_{\hbar}\right) \leq h_{m}^{+}\left(\phi_{\hbar}\right)+h_{n_{0}}^{+}\left(U^{m \hbar} \phi_{\hbar}\right)+R_{n_{0}}(\hbar), \\
& h_{n_{0}+m}^{-}\left(\phi_{\hbar}\right) \leq h_{n_{0}}^{-}\left(\phi_{\hbar}\right)+h_{m}^{-}\left(U^{n_{0} \hbar} \phi_{\hbar}\right)+R_{n_{0}}(\hbar) .
\end{aligned}
$$

Let $n_{0} \in \mathbb{N}$ be fixed and $n=n_{\mathrm{Ehr}}(\hbar, E, \varepsilon)$. Using the Euclidean division $n=q n_{0}+r$, with $r<n_{0}$, Proposition 6.4 implies that for $\hbar$ small enough,

$$
\frac{h_{n}^{+}\left(\phi_{\hbar}\right)}{n} \leq \frac{\sum_{k=0}^{q-1} h_{n_{0}}^{+}\left(U^{k n_{0} \hbar} \phi_{\hbar}\right)}{q n_{0}}+\frac{h_{r}^{+}\left(U^{q n_{0} \hbar} \phi_{\hbar}\right)}{n}+\frac{R_{n_{0}}(\hbar)}{n_{0}}
$$


and

$$
\frac{h_{n}^{-}\left(\phi_{\hbar}\right)}{n} \leq \frac{\sum_{k=0}^{q-1} h_{n_{0}}^{-}\left(U^{\left(r+k n_{0}\right) \hbar} \phi_{\hbar}\right)}{q n_{0}}+\frac{h_{r}^{-}\left(U^{r \hbar} \phi_{\hbar}\right)}{n}+\frac{R_{n_{0}}(\hbar)}{n_{0}} .
$$

Note that $h_{r}^{+}\left(U^{q n_{0} \hbar} \phi_{\hbar}\right)+h_{r}^{-}\left(U^{r \hbar} \phi_{\hbar}\right)$ stays uniformly bounded (by $\left.\log n_{0}\right)$ when $\hbar \rightarrow 0$. Combining the subadditivity property with Corollary 6.3 , we find that

$$
\begin{aligned}
& \frac{\sum_{k=0}^{q-1}\left(h_{n_{0}}^{+}\left(U^{k n_{0} \hbar} U^{n \hbar} \phi_{\hbar}\right)+h_{n_{0}}^{-}\left(U^{\left(r+k n_{0}\right) \hbar} \phi_{\hbar}\right)\right)}{2 q n_{0}} \\
& \quad \geq(d-1) \sqrt{E-\varepsilon}-\frac{(d-1) \sqrt{E+\varepsilon}}{2(1-\delta)}-\frac{R_{n_{0}}(\hbar)}{n_{0}}+\mathcal{O}_{n_{0}}(1 / n)
\end{aligned}
$$

for $n=n_{\mathrm{Ehr}}(\hbar, E, \varepsilon)$.

6d. The conclusion. The interval $\left[E_{1}, E_{2}\right]$ is fixed. Consider $E$ in $\left[E_{1}, E_{2}\right]$ and a sequence of normalized states $\left(u_{\hbar}\right)_{\hbar \rightarrow 0}$ that satisfies (7). We may assume without loss of generality that $\mathbb{1}_{\left[E_{1}, E_{2}\right]}\left(-\hbar^{2} \Delta\right) u_{\hbar}=$ $u_{\hbar}$ (since the semiclassical limits associated with $u_{\hbar}$ and $\mathbb{1}_{\left[E_{1}, E_{2}\right]}\left(-\hbar^{2} \Delta\right) u_{\hbar}$ will be the same). We fix a function $\chi \in \mathscr{C}_{o}^{\infty}(\mathbb{R})$, supported in $[-1,1]$ such that $\sum_{k \in \mathbb{Z}} \chi^{2}(x-k) \equiv 1$. For $N \in \mathbb{N}$, we write

$$
\varepsilon=\frac{E_{2}-E_{1}}{N} \text { and } \chi_{j}(x)=\chi\left(\frac{x-E_{1}-j \varepsilon}{\varepsilon}\right) \text { for } j=0, \ldots, N .
$$

We have $u_{\hbar}=\sum_{j=0}^{N} \chi_{j}^{2}\left(-\hbar^{2} \Delta\right) u_{\hbar}$ and thus $\left\|u_{\hbar}\right\|^{2}=\sum_{j=0}^{N}\left\|\chi_{j}\left(-\hbar^{2} \Delta\right) u_{\hbar}\right\|^{2}$. We will write $u_{j}=$ $\chi_{j}\left(-\hbar^{2} \Delta\right) u_{\hbar}$ and $\tilde{u}_{j}=u_{j} /\left\|u_{j}\right\|$. For $t \in \mathbb{R}$, we apply (28) to $\phi_{\hbar}=U^{t} \tilde{u}_{j}$ and obtain

$$
\begin{aligned}
& \frac{\sum_{k=0}^{q-1}\left(h_{n_{0}}^{+}\left(U^{k n_{0} \hbar} U^{n \hbar} U^{t} \tilde{u}_{j}\right)+h_{n_{0}}^{-}\left(U^{\left(r+k n_{0}\right) \hbar} U^{t} \tilde{u}_{j}\right)\right)}{2 q n_{0}} \\
& \geq(d-1) \sqrt{E_{1}+(j-1) \varepsilon}-\frac{(d-1)}{2(1-\delta)} \sqrt{E_{1}+(j+1) \varepsilon}-\frac{R_{n_{0}}(\hbar)}{n_{0}}+\mathcal{O}_{n_{0}}(1 /|\log \hbar|) .
\end{aligned}
$$

If we multiply by $\theta(t)$ (satisfying $\theta \in L^{1}\left(\mathbb{R}, \mathbb{R}_{+}\right)$and $\int \theta=1$ ), integrate with respect to $t$, and take into account the fact that $\left(k n_{0}+r\right) \hbar \rightarrow 0$ and $n \hbar \rightarrow 0$, we find that

$$
\int \theta(t) \frac{h_{n_{0}}^{+}\left(U^{t} \tilde{u}_{j}\right)+h_{n_{0}}^{-}\left(U^{t} \tilde{u}_{j}\right)}{2 n_{0}} d t \geq(d-1) \sqrt{E_{1}+(j-1) \varepsilon}-\frac{(d-1)}{2(1-\delta)} \sqrt{E_{1}+(j+1) \varepsilon}+o_{n_{0}}(1) .
$$

This yields that

$$
\begin{aligned}
\sum_{j=0}^{N}\left\|u_{j}\right\|^{2} \int \theta(t) \frac{h_{n_{0}}^{+}\left(U^{t} \tilde{u}_{j}\right)+h_{n_{0}}^{-}\left(U^{t} \tilde{u}_{j}\right)}{2 n_{0}} d t \\
\quad \geq \sum_{j=0}^{N}\left\|u_{j}\right\|^{2}\left[(d-1) \sqrt{E_{1}+(j-1) \varepsilon}-\frac{(d-1)}{2(1-\delta)} \sqrt{E_{1}+(j+1) \varepsilon}\right]+o_{n_{0}}(1)
\end{aligned}
$$


We define the averaged entropy

$$
\begin{aligned}
& h_{n}^{-}(\phi, \theta)=-\sum_{|\alpha|=n}\left(\int \theta(t)\left\|\pi_{\alpha}^{*} U^{t} \phi\right\|^{2} d t\right) \log \left(\int \theta(t)\left\|\pi_{\alpha}^{*} U^{t} \phi\right\|^{2} d t\right), \\
& h_{n}^{+}(\phi, \theta)=-\sum_{|\alpha|=n}\left(\int \theta(t)\left\|\pi_{\alpha} U^{t} \phi\right\|^{2} d t\right) \log \left(\int \theta(t)\left\|\pi_{\alpha} U^{t} \phi\right\|^{2} d t\right) .
\end{aligned}
$$

Using the concavity of $x \mapsto-x \log x$, (31) implies

$$
\begin{aligned}
& \sum_{j=0}^{N}\left\|u_{j}\right\|^{2} \frac{h_{n_{0}}^{+}\left(\tilde{u}_{j}, \theta\right)+}{2 n_{0}}-\left(\tilde{u}_{j}, \theta\right) \\
& \quad \geq \sum_{j=0}^{N}\left\|u_{j}\right\|^{2}\left[(d-1) \sqrt{E_{1}+(j-1) \varepsilon}-\frac{(d-1)}{2(1-\delta)} \sqrt{E_{1}+(j+1) \varepsilon}\right]+o_{n_{0}}(1)
\end{aligned}
$$

We can now take the limit $\hbar \rightarrow 0$. If the semiclassical measure associated with the family $\left(U^{t} u_{\hbar}\right)$ decomposes as $\mu_{t}=\int \mu_{t, E} d v(E)$, then $\left\|u_{j}\right\|^{2}$ converges to $\int \chi_{j}^{2}(E) d v(E)$. On the left side of (34), $h_{n_{0}}^{+}\left(\tilde{u}_{j}, \theta\right)$ and $h_{n_{0}}^{-}\left(\tilde{u}_{j}, \theta\right)$ both converge to

$$
\sum_{|\alpha|=n_{0}} \eta\left(\frac{1}{\int \chi_{j}^{2}(E) d \nu(E)} \int \theta(t) \chi_{j}^{2}(E) \mu_{t, E}\left(\left(P_{\alpha_{n-1}}^{2} \circ g^{n-1}\right) \cdots\left(P_{\alpha_{1}}^{2} \circ g^{1}\right) P_{\alpha_{0}}^{2}\right) d \nu(E) d t\right)
$$

where $\eta(x)=-x \log x$.

Then, we let $n_{0} \rightarrow+\infty$, which allows to go from the previous quantity to the Kolmogorov-Sinai entropy $h_{\mathrm{KS}}$; for this step, details can be found in [Anantharaman and Nonnenmacher 2007, Section 2.2.8]. This gives us the inequality

$$
\begin{aligned}
\sum_{j=0}^{N} \int \chi_{j}^{2}(E) d \nu(E) & h_{\mathrm{KS}}\left(\frac{1}{\int \chi_{j}^{2}(E) d \nu(E)} \int \theta(t) \chi_{j}^{2}(E) \mu_{t, E} d \nu(E) d t\right) \\
& \geq \sum_{j=0}^{N}\left[(d-1) \sqrt{E_{1}+(j-1) \varepsilon}-\frac{(d-1)}{2(1-\delta)} \sqrt{E_{1}+(j+1) \varepsilon}\right] \int \chi_{j}^{2}(E) d v(E)
\end{aligned}
$$

At this stage, we use the fact that $h_{\mathrm{KS}}$ is affine and derive that

$$
\int \theta(t)\left(h_{\mathrm{KS}}\left(\mu_{t, E}\right)-\sum_{j=0}^{N} \chi_{j}^{2}(E)\left[(d-1) \sqrt{E_{1}+(j-1) \varepsilon}-\frac{(d-1)}{2(1-\delta)} \sqrt{E_{1}+(j+1) \varepsilon}\right]\right) d \nu(E) d t \geq 0
$$

Finally, we can take the limit $N \rightarrow+\infty$, to obtain

$$
\int \theta(t)\left(h_{\mathrm{KS}}\left(\mu_{t, E}\right)-\frac{d-1}{2} \sqrt{E}\right) d v(E) d t \geq 0 .
$$


If we use the same argument, replacing $u_{\hbar}$ by $f\left(-\hbar^{2} \Delta\right) u_{\hbar}$ (where $f$ is a smooth function on $\left[E_{1}, E_{2}\right]$ such that $\left.\int f^{2}(E) d v(E)=1\right)$, we obtain by the same argument

$$
\int \theta(t) f^{2}(E)\left(h_{\mathrm{KS}}\left(\mu_{t, E}\right)-\frac{d-1}{2} \sqrt{E}\right) d v(E) d t \geq 0
$$

this holds for all $\theta$ in $L^{1}\left(\mathbb{R}, \mathbb{R}_{+}\right)$such that $\int \theta=1$ and $f$ in $\mathscr{C}_{o}^{\infty}\left(\mathbb{R}_{+}, \mathbb{R}\right)$ such that $\int f^{2}(E) d \nu(E)=1$. As a consequence, one has for Leb $\otimes v$-almost every $(t, E)$,

$$
h_{\mathrm{KS}}\left(\mu_{t, E}\right) \geq \frac{d-1}{2} \sqrt{E} \text {. }
$$

Remark 10. If one wants to consider the microlocal setting (see Remark 4) where one uses Op instead $_{1}$ of $\mathrm{Op}_{\hbar}$, one introduces a partition of unity based on the Paley-Littlewood decomposition. For a fixed $\epsilon>0$, arbitrarily small, one introduces a smooth function $\psi_{\epsilon}$ on $\mathbb{R}_{+}$satisfying $\psi_{\epsilon}(E)=1$ for $0 \leq E \leq 2^{-\epsilon}$ and $\psi_{\epsilon}(E)=0$ for $E \geq 1$. Then, one can define $\varphi_{\epsilon}(E)=\psi_{\epsilon}\left(E / 2^{\epsilon}\right)-\psi_{\epsilon}(E)$ and verify that

$$
1=\psi_{\epsilon}(E)+\sum_{j \geq 0} \varphi_{\epsilon}\left(2^{-j \epsilon} E\right) .
$$

We stress that for every $j \geq 0$, the cutoff function $\varphi_{\epsilon}\left(2^{-j \epsilon} E\right)$ is compactly supported in $\left[2^{\epsilon(j-1)}, 2^{\epsilon(j+1)}\right]$. On the energy window $E \in\left[2^{\epsilon(j-1)}, 2^{\epsilon(j+1)}\right]$, one can adapt the proof above, doing the change of variable $\xi \rightsquigarrow 2^{-\epsilon j} \xi$, and using the relation $\mathrm{Op}_{1}\left(a\left(x, 2^{-\epsilon j} \xi\right)\right)=\mathrm{Op}_{2^{-\epsilon j}}(a(x, \xi))$. One then copies the steps of Section 6 , using $\hbar_{j}=2^{-\epsilon j}$ as the effective Planck constant, and taking $\chi_{j}(E)=\varphi_{\epsilon}^{1 / 2}\left(2^{-j \epsilon} E\right)$ in Section 6d.

\section{From entropy estimates to observability}

In this section, we explain how we can go from the entropy estimates of Theorem 2.4 to the observability estimate of Theorem 2.5. According to Lebeau [1992], it suffices to prove the following weak observability result to deduce Theorem 2.5:

Theorem 7.1. Under the assumptions of Theorem 2.5, for all $T>0$, there exists $C_{T, a}>0$ such that

$$
\|u\|_{L^{2}(M)}^{2} \leq C_{T, a}\left(\int_{0}^{T}\left\|a e^{\imath t \Delta / 2} u\right\|_{L^{2}(M)}^{2} d t+\|u\|_{H^{-1}(M)}^{2}\right) \quad \text { for all } u \text { in } L^{2}(M) .
$$

For the sake of completeness, we briefly recall the argument of Lebeau to deduce observability from a weak observability estimate at time $T$. First, for $T^{\prime}>T$, we introduce the subspace

$$
N\left(T^{\prime}\right):=\left\{\varphi \in L^{2}(M): a(x)\left(e^{l t \Delta} \varphi\right)(x)=0 \text { for all } 0 \leq t \leq T^{\prime}\right\}
$$

From weak observability and the compactness of the injection $L^{2} \subset H^{-1}$, we can deduce that for $T^{\prime}>T$, this subspace is finite-dimensional. One can also verify that $\Delta \varphi$ belongs to $N\left(T^{\prime \prime}\right)$ for every $T<T^{\prime \prime}<T^{\prime}$ and every $\varphi$ in $N\left(T^{\prime}\right)$ (by taking the limit of the sequence $\left(e^{l \epsilon \Delta} \varphi-\varphi\right) / \epsilon$, which belongs to $N\left(T^{\prime \prime}\right)$ for $\epsilon$ small enough, and is bounded in $\left.H^{-2}(M)\right)$.

This implies that $\Delta$ is an operator from the finite-dimensional subspace $N\left(T^{\prime}\right)$ into itself. As $a$ is nontrivial, one can deduce the existence of an eigenfunction of the Laplacian that is equal to 0 on a 
nonempty open set. By the Aronszajn-Cordes theorem [Hörmander 1985, Section 17.2], this eigenfunction is necessarily 0 and the subspace $N\left(T^{\prime}\right)$ is reduced to $\{0\}$. By contradiction, we can finally deduce that observability holds for $T^{\prime}>T$.

To prove Theorem 7.1, we proceed by contradiction and make the assumption that there exists a sequence of normalized vectors $\left(u_{n}\right)_{n \in \mathbb{N}}$ in $L^{2}(M)$ and $T>0$ such that

$$
\lim _{n \rightarrow+\infty}\left(\int_{0}^{T}\left\|a e^{l t \Delta / 2} u_{n}\right\|_{L^{2}(M)}^{2} d t+\left\|u_{n}\right\|_{H^{-1}(M)}^{2}\right)=0 .
$$

This implies that $u_{n}$ converges to 0 , weakly in $L^{2}$. For every $t$ in $\mathbb{R}$, we introduce the "distribution"

$$
\mu_{n}(t)(b):=\left\langle u_{n} \mid e^{-l t \Delta / 2} \mathrm{Op}_{1}(b) e^{l t \Delta / 2} u_{n}\right\rangle_{L^{2}(M)},
$$

defined for all $b \in \mathscr{S}_{0}$. The map $t \mapsto \mu_{n}(t)$ belongs to $L^{\infty}\left(\mathbb{R}, \mathscr{Y}_{0}^{\prime}\right)$. Thus, there exists a subsequence $\left(u_{n_{k}}\right)_{k}$ and $\mu$ in $L^{\infty}\left(\mathbb{R}, \mathscr{Y}_{0}^{\prime}\right)$ such that

$$
\int_{\mathbb{R} \times \widehat{T^{*} M}} \theta(t) b(x, \xi) \mu_{n_{k}}(t)(d x, d \xi) d t \underset{k \rightarrow+\infty}{\longrightarrow} \int_{\mathbb{R} \times \widehat{T^{*} M}} \theta(t) b(x, \xi) \mu(t)(d x, d \xi) d t
$$

for all $\theta \in L^{1}(\mathbb{R})$ and $b \in \mathscr{Y}_{0}$. As $u_{n}$ converges weakly to 0 , each $\mu(t)$ is actually supported at infinity, and may thus be identified with a probability measure on the unit sphere bundle $S^{*} M$, invariant under the geodesic flow (see Remark 4).

From Theorem 2.4 and Remark 4, we know that $h_{\mathrm{KS}}(\mu(t)) \geq \frac{1}{2}(d-1)$ for almost every $t$ in $\mathbb{R}$. We will now use the fact that the topological entropy of $K_{a}$ is less than $\frac{1}{2}(d-1)$, that is,

$$
h_{\text {top }}\left(K_{a},\left(g^{\tau}\right)\right):=\sup _{\mu \in \mathcal{M}\left(S^{*} M, g^{\tau}\right)}\left\{h_{\mathrm{KS}}(\mu): \mu\left(K_{a}\right)=1\right\}<\frac{1}{2}(d-1) .
$$

Using property (37), we know that $\int_{S^{*} M \times[0, T]} a^{2}(x, \xi) \mu(t)(d x, d \xi) d t=0$. In particular, this implies that $\mu(t)\left(S^{*} M \backslash K_{a}\right)=0$ for almost every $t$ in [0,T] (as $\mu(t)$ is $g^{\tau}$-invariant), leading to a contradiction.

\section{Appendix A. Pseudodifferential calculus on a manifold}

In this section, we recall some facts of pseudodifferential calculus; details can be found in [Zworski 2012]. We define on $\mathbb{R}^{2 d}$ the following class of (semiclassical) symbols:

$$
S^{m, k}\left(\mathbb{R}^{2 d}\right):=\left\{a=a_{\hbar} \in C^{\infty}\left(\mathbb{R}^{2 d}\right):\left|\partial_{x}^{\alpha} \partial_{\xi}^{\beta} a\right| \leq C_{\alpha, \beta} \hbar^{-k}\langle\xi\rangle^{m-|\beta|}\right.
$$

for all $K \subset \mathbb{R}^{d}$ compact, $\alpha, \beta$, some $C_{\alpha, \beta}$, and all $\left.(x, \xi) \in K \times \mathbb{R}^{d}\right\}$.

Let $M$ be a smooth compact Riemannian $d$-manifold without boundary. Consider a finite smooth atlas $\left(f_{l}, V_{l}\right)$ of $M$, where each $f_{l}$ is a smooth diffeomorphism from the open subset $V_{l} \subset M$ to a bounded open set $W_{l} \subset \mathbb{R}^{d}$. To each $f_{l}$ corresponds a pull-back $f_{l}^{*}: C^{\infty}\left(W_{l}\right) \rightarrow C^{\infty}\left(V_{l}\right)$ and a canonical map $\tilde{f}_{l}$ from $T^{*} V_{l}$ to $T^{*} W_{l}$ :

$$
\tilde{f}_{l}:(x, \xi) \mapsto\left(f_{l}(x),\left(D f_{l}(x)^{-1}\right)^{T} \xi\right)
$$


Consider now a smooth locally finite partition of identity $\left(\phi_{l}\right)$ adapted to the previous atlas $\left(f_{l}, V_{l}\right)$. That means $\sum_{l} \phi_{l}=1$ and $\phi_{l} \in \mathscr{C}_{o}^{\infty}\left(V_{l}\right)$. Then, any observable $a$ in $C^{\infty}\left(T^{*} M\right)$ can be decomposed as $a=\sum_{l} a_{l}$, where $a_{l}=a \phi_{l}$. Each $a_{l}$ belongs to $C^{\infty}\left(T^{*} V_{l}\right)$ and can be pushed to a function $\tilde{a}_{l}=$ $\left(\tilde{f}_{l}^{-1}\right)^{*} a_{l} \in C^{\infty}\left(T^{*} W_{l}\right)$. As in [Zworski 2012], define a class of symbols of order $m$ and index $k$ by

$$
S^{m, k}\left(T^{*} M\right):=\left\{a=a_{\hbar} \in C^{\infty}\left(T^{*} M\right):\left|\partial_{x}^{\alpha} \partial_{\xi}^{\beta} a\right| \leq C_{\alpha, \beta} \hbar^{-k}\langle\xi\rangle^{m-|\beta|} \text { for all } \alpha, \beta \text { and some } C_{\alpha, \beta}\right\} .
$$

Then, for $a \in S^{m, k}\left(T^{*} M\right)$ and for each $l$, one can associate to the symbol $\tilde{a}_{l} \in S^{m, k}\left(\mathbb{R}^{2 d}\right)$ the standard Weyl quantization:

$$
\mathrm{Op}_{\hbar}^{w}\left(\tilde{a}_{l}\right) u(x):=\frac{1}{(2 \pi \hbar)^{d}} \int_{\mathbb{R}^{2 d}} e^{(l / \hbar)\langle x-y, \xi\rangle} \tilde{a}_{l}\left(\frac{x+y}{2}, \xi ; \hbar\right) u(y) d y d \xi,
$$

where $u \in \mathscr{C}_{o}^{\infty}\left(\mathbb{R}^{d}\right)$. Consider now a smooth cutoff $\psi_{l} \in \mathscr{C}_{c}^{\infty}\left(V_{l}\right)$ such that $\psi_{l}=1$ close to the support of $\phi_{l}$. A quantization of $a \in S^{m, k}\left(T^{*} M\right)$ is then defined by

$$
\mathrm{Op}_{\hbar}(a)(u):=\sum_{l} \psi_{l} \times\left(f_{l}^{*} \mathrm{Op}_{\hbar}^{w}\left(\tilde{a}_{l}\right)\left(f_{l}^{-1}\right)^{*}\right)\left(\psi_{l} \times u\right),
$$

where $u \in \mathscr{C}^{\infty}(M)$. According to the appendix of [Zworski 2012], the quantization procedure $\mathrm{Op}_{\hbar}$ sends $S^{m, k}\left(T^{*} M\right)$ onto the space of pseudodifferential operators of order $m$ and of index $k$, denoted $\Psi^{m, k}(M)$. It can be shown that the dependence in the cutoffs $\phi_{l}$ and $\psi_{l}$ only appears at order 2 in $\hbar$ and the principal symbol map $\sigma_{0}: \Psi^{m, k}(M) \rightarrow S^{m, k} / S^{m, k-1}\left(T^{*} M\right)$ is then intrinsically defined.

At various places in this paper, a larger class of symbols should be considered, as in [Dimassi and Sjöstrand 1999] or [Zworski 2012]. For $0 \leq v<1 / 2$,

$$
S_{v}^{m, k}\left(T^{*} M\right)=\left\{a=a_{\hbar} \in \mathscr{C}^{\infty}\left(T^{*} M\right):\left|\partial_{x}^{\alpha} \partial_{\xi}^{\beta} a\right| \leq C_{\alpha, \beta} \hbar^{-k-v|\alpha+\beta|}\langle\xi\rangle^{m-|\beta|} \text { for all } \alpha, \beta \text { and some } C_{\alpha, \beta}\right\} .
$$

Results of [Dimassi and Sjöstrand 1999] can be applied to this new class of symbols. For example, if $M$ is compact, a symbol of $S_{v}^{0,0}$ gives a bounded operator on $L^{2}(M)$ (with norm independent of $\hbar \leq 1$ ).

Even if the Weyl procedure is a natural choice to quantize an observable $a$ on $\mathbb{R}^{2 d}$, it is sometimes preferable to use a quantization that also satisfies the property that $\mathrm{Op}_{\hbar}(a) \geq 0$ if $a \geq 0$ (such a quantization procedure is said to be positive). This can be achieved using to the anti-Wick procedure; see [Helffer et al. 1987]. For $a$ in $S_{v}^{0,0}\left(\mathbb{R}^{2 d}\right)$ that coincides with a function on $\mathbb{R}^{d}$ outside a compact subset of $T^{*} \mathbb{R}^{d}$, one has

$$
\left\|\mathrm{Op}_{\hbar}^{w}(a)-\mathrm{Op}_{\hbar}^{A W}(a)\right\|_{L^{2}} \leq C \sum_{|\alpha| \leq D} \hbar^{(|\alpha|+1) / 2}\left\|\partial^{\alpha} a\right\|_{\infty},
$$

where $C$ and $D$ are some positive constants that depend only on the dimension $d$. To get a positive procedure of quantization on a manifold, one replaces in definition (39) the Weyl quantization by the anti-Wick one. We will denote by $\mathrm{Op}_{\hbar}^{+}(a)$ this new choice of quantization, which is well defined for every element in $S_{v}^{0,0}\left(T^{*} M\right)$ of the form $b(x)+c(x, \xi)$, where $b$ belongs to $S_{v}^{0,0}\left(T^{*} M\right)$ and $c$ belongs to $\mathscr{C}_{o}^{\infty}\left(T^{*} M\right) \cap S_{v}^{0,0}\left(T^{*} M\right)$. We underline the fact that $\mathrm{Op}_{\hbar}^{+}(1)=\operatorname{Id}_{L^{2}(M)}$. 


\section{References}

[Anantharaman 2008] N. Anantharaman, "Entropy and the localization of eigenfunctions", Ann. of Math. (2) 168:2 (2008), 435-475. MR 2011g:35076 Zbl 1175.35036

[Anantharaman 2011] N. Anantharaman, "Exponential decay for products of Fourier integral operators", Methods Appl. Anal. 18:2 (2011), 165-181. MR 2847483

[Anantharaman and Macià 2011] N. Anantharaman and F. Macià, "Semiclassical measures for the Schrödinger equation on the torus", preprint, 2011. arXiv 1005.0296

[Anantharaman and Nonnenmacher 2007] N. Anantharaman and S. Nonnenmacher, "Half-delocalization of eigenfunctions for the Laplacian on an Anosov manifold", Ann. Inst. Fourier (Grenoble) 57:7 (2007), 2465-2523. MR 2009m:81076 Zbl 1145.81033

[Anantharaman et al. 2009] N. Anantharaman, H. Koch, and S. Nonnenmacher, "Entropy of eigenfunctions", pp. 1-22 in New trends in mathematical physics (Rio de Janeiro, 2006), edited by V. Sidoravičius, Springer, Dordrecht, 2009. Zbl 1175.81118 arXiv 0704.1564

[Barreira and Wolf 2007] L. Barreira and C. Wolf, "Dimension and ergodic decompositions for hyperbolic flows", Discrete Contin. Dyn. Syst. 17:1 (2007), 201-212. MR 2007m:37050 Zbl 1144.37012

[Bouzouina and Robert 2002] A. Bouzouina and D. Robert, "Uniform semiclassical estimates for the propagation of quantum observables", Duke Math. J. 111:2 (2002), 223-252. MR 2003b:81049 Zbl 1069.35061

[Bowen and Ruelle 1975] R. Bowen and D. Ruelle, "The ergodic theory of Axiom A flows", Invent. Math. 29:3 (1975), 181202. MR 52 \#1786 Zbl 0311.58010

[Colin de Verdière 1985] Y. Colin de Verdière, "Ergodicité et fonctions propres du Laplacien", Comm. Math. Phys. 102:3 (1985), 497-502. MR 87d:58145 Zbl 0592.58050

[Dimassi and Sjöstrand 1999] M. Dimassi and J. Sjöstrand, Spectral asymptotics in the semi-classical limit, London Mathematical Society Lecture Note Series 268, Cambridge University Press, 1999. MR 2001b:35237 Zbl 0926.35002

[Duistermaat and Guillemin 1975] J. J. Duistermaat and V. W. Guillemin, "The spectrum of positive elliptic operators and periodic bicharacteristics", Invent. Math. 29:1 (1975), 39-79. MR 53 \#9307 Zbl 0307.35071

[Eckhardt et al. 1995] B. Eckhardt, S. Fishman, J. P. Keating, O. Agam, J. Main, and K. Muller, "Approach to ergodicity in quantum wave functions", Phys. Rev. E 52:6 (1995), 5893-5903.

[Feingold and Peres 1986] M. Feingold and A. Peres, "Distribution of matrix elements of chaotic systems", Phys. Rev. A (3) 34:1 (1986), 591-595. MR 87i:81055

[Gérard 1991] P. Gérard, "Microlocal defect measures”, Comm. Partial Differential Equations 16:11 (1991), 1761-1794. MR 92k:35027 Zbl 0770.35001

[Helffer et al. 1987] B. Helffer, A. Martinez, and D. Robert, "Ergodicité et limite semi-classique", Comm. Math. Phys. 109:2 (1987), 313-326. MR 88e:81029 Zbl 0624.58039

[Hörmander 1985] L. Hörmander, The analysis of linear partial differential operators, III: Pseudo-differential operators, Grundlehren der Mathematischen Wissenschaften 274, Springer, Berlin, 1985. MR 87d:35002a Zbl 0601.35001

[Katok and Hasselblatt 1995] A. Katok and B. Hasselblatt, Introduction to the modern theory of dynamical systems, Encyclopedia of Mathematics and its Applications 54, Cambridge University Press, 1995. MR 96c:58055 Zbl 0878.58020

[Kifer 1992] Y. Kifer, "Averaging in dynamical systems and large deviations", Invent. Math. 110:2 (1992), 337-370. MR 93m: 60118 Zbl 0791.58072

[Lebeau 1992] G. Lebeau, “Contrôle de l'équation de Schrödinger”, J. Math. Pures Appl. (9) 71:3 (1992), 267-291. MR 93i: 35018 Zbl 0838.35013

[Ledrappier and Young 1985] F. Ledrappier and L.-S. Young, "The metric entropy of diffeomorphisms, I: Characterization of measures satisfying Pesin's entropy formula", Ann. of Math. (2) 122:3 (1985), 509-539. MR 87i:58101a Zbl 0605.58028

[Maassen and Uffink 1988] H. Maassen and J. B. M. Uffink, "Generalized entropic uncertainty relations", Phys. Rev. Lett. 60:12 (1988), 1103-1106. MR 89f:81027 
[Macià 2009] F. Macià, "Semiclassical measures and the Schrödinger flow on Riemannian manifolds", Nonlinearity 22:5 (2009), 1003-1020. MR 2010i:58032 Zbl 1166.81020

[Macià 2010] F. Macià, "High-frequency propagation for the Schrödinger equation on the torus", J. Funct. Anal. 258:3 (2010), 933-955. MR 2011b:81143 Zbl 1180.35438

[Parry and Pollicott 1990] W. Parry and M. Pollicott, Zeta functions and the periodic orbit structure of hyperbolic dynamics, Astérisque 187-188, Société Mathématique de France, Montrouge, 1990. MR 92f:58141 Zbl 0726.58003

[Pesin 1977] Y. B. Pesin, "Characteristic Lyapunov exponents, and smooth ergodic theory", Uspehi Mat. Nauk 32:4 (196) (1977), 55-112. In Russian; translated in Russ. Math. Surv. 32:4 (1977), 55-114. MR 57 \#6667 Zbl 0359.58010

[Pesin 1997] Y. B. Pesin, Dimension theory in dynamical systems: contemporary views and applications, University of Chicago Press, Chicago, IL, 1997. MR 99b:58003 Zbl 0895.58033

[Pesin and Sadovskaya 2001] Y. B. Pesin and V. Sadovskaya, "Multifractal analysis of conformal Axiom A flows", Comm. Math. Phys. 216:2 (2001), 277-312. MR 2002g:37035 Zbl 0992.37023

[Rees 1981] M. Rees, "An alternative approach to the ergodic theory of measured foliations on surfaces", Ergodic Theory Dynamical Systems 1:4 (1981), 461-488. MR 84c:58065 Zbl 0539.58018

[Rivière 2009] G. Rivière, Délocalisation des mesures semi-classiques pour des systèmes dynamiques chaotiques, thesis, Centre de Mathématiques Laurent Schwartz, Palaiseau, 2009, available at http://tel.archives-ouvertes.fr/tel-00437912.

[Rivière 2010] G. Rivière, "Entropy of semiclassical measures in dimension 2", Duke Math. J. 155:2 (2010), 271-336. MR 2012a:58056 Zbl 1230.37048

[Ruelle 1976] D. Ruelle, "Generalized zeta-functions for Axiom A basic sets”, Bull. Amer. Math. Soc. 82:1 (1976), 153-156. MR 53 \#4146 Zbl 0316.58016

[Ruelle 1978] D. Ruelle, "An inequality for the entropy of differentiable maps", Bol. Soc. Brasil. Mat. 9:1 (1978), 83-87. MR 80f:58026 Zbl 0432.58013

[Schubert 2006] R. Schubert, “Upper bounds on the rate of quantum ergodicity”, Ann. Henri Poincaré 7:6 (2006), 1085-1098. MR 2008a:81075 Zbl 1099.81032

[Shnirelman 1974] A. I. Shnirelman, "Ergodic properties of eigenfunctions", Uspehi Mat. Nauk 29:6 (180) (1974), 181-182. In Russian. MR 53 \#6648 Zbl 0324.58020

[Sogge and Zelditch 2002] C. D. Sogge and S. Zelditch, "Riemannian manifolds with maximal eigenfunction growth", Duke Math. J. 114:3 (2002), 387-437. MR 2004b:58053 Zbl 1018.58010

[Walters 1982] P. Walters, An introduction to ergodic theory, Graduate Texts in Mathematics 79, Springer, New York, 1982. MR 84e:28017 Zbl 0475.28009

[Zelditch 1987] S. Zelditch, "Uniform distribution of eigenfunctions on compact hyperbolic surfaces", Duke Math. J. 55:4 (1987), 919-941. MR 89d:58129 Zbl 0643.58029

[Zelditch 1994] S. Zelditch, "On the rate of quantum ergodicity, I: Upper bounds", Comm. Math. Phys. 160:1 (1994), 81-92. MR 95f:58084 Zbl 0788.58043

[Zworski 2012] M. Zworski, Semiclassical analysis, American Mathematical Society, Providence, RI, 2012.

Received 28 Jul 2010. Revised 27 Aug 2011. Accepted 31 Aug 2011.

NALINI ANANTHARAMAN: nalini.anantharaman@math.u-psud.fr

Laboratoire de Mathématiques (UMR 8628), Université Paris-Sud XI, Bâtiment 425, 91405 Orsay Cedex, France

GABRIEL RIVIÈRE: gabriel.riviere@polytechnique.edu

Laboratoire Paul Painlevé (UMR 8524), Université Lille 1, Bâtiment M3, 59655 Villeneuve d'Ascq Cedex, France 


\title{
Analysis \& PDE
}

\author{
msp.berkeley.edu/apde
}

EDITORS

EDITOR-IN-CHIEF

Maciej Zworski

University of California

Berkeley, USA

BOARD OF EDITORS

\begin{tabular}{|c|c|c|c|}
\hline Michael Aizenman & $\begin{array}{l}\text { Princeton University, USA } \\
\text { aizenman@math.princeton.edu }\end{array}$ & Nicolas Burq & $\begin{array}{l}\text { Université Paris-Sud 11, France } \\
\text { nicolas.burq@math.u-psud.fr }\end{array}$ \\
\hline Luis A. Caffarelli & $\begin{array}{l}\text { University of Texas, USA } \\
\text { caffarel@math.utexas.edu }\end{array}$ & un-Yung Alice Chang & $\begin{array}{l}\text { Princeton University, USA } \\
\text { chang@math.princeton.edu }\end{array}$ \\
\hline Michael Christ & $\begin{array}{l}\text { University of California, Berkeley, USA } \\
\text { mchrist@math.berkeley.edu }\end{array}$ & Charles Fefferman & $\begin{array}{l}\text { Princeton University, USA } \\
\text { cf@math.princeton.edu }\end{array}$ \\
\hline Ursula Hamenstaedt & $\begin{array}{l}\text { Universität Bonn, Germany } \\
\text { ursula@math.uni-bonn.de }\end{array}$ & Nigel Higson & $\begin{array}{l}\text { Pennsylvania State Univesity, USA } \\
\text { higson@ math.psu.edu }\end{array}$ \\
\hline Vaughan Jones & $\begin{array}{l}\text { University of California, Berkeley, USA } \\
\text { vfr@math.berkeley.edu }\end{array}$ & Herbert Koch & $\begin{array}{l}\text { Universität Bonn, Germany } \\
\text { koch@math.uni-bonn.de }\end{array}$ \\
\hline Izabella Laba & $\begin{array}{l}\text { University of British Columbia, Canada } \\
\text { ilaba@math.ubc.ca }\end{array}$ & Gilles Lebeau & $\begin{array}{l}\text { Université de Nice Sophia Antipolis, France } \\
\text { lebeau@unice.fr }\end{array}$ \\
\hline László Lempert & $\begin{array}{l}\text { Purdue University, USA } \\
\text { lempert@math.purdue.edu }\end{array}$ & Richard B. Melrose & $\begin{array}{l}\text { Massachussets Institute of Technology, USA } \\
\text { rbm@math.mit.edu }\end{array}$ \\
\hline Frank Merle & $\begin{array}{l}\text { Université de Cergy-Pontoise, France } \\
\text { Frank.Merle@u-cergy.fr }\end{array}$ & William Minicozzi II & $\begin{array}{l}\text { Johns Hopkins University, USA } \\
\text { minicozz@ math.jhu.edu }\end{array}$ \\
\hline Werner Müller & $\begin{array}{l}\text { Universität Bonn, Germany } \\
\text { mueller@math.uni-bonn.de }\end{array}$ & Yuval Peres & $\begin{array}{l}\text { University of California, Berkeley, USA } \\
\text { peres@stat.berkeley.edu }\end{array}$ \\
\hline Gilles Pisier & $\begin{array}{l}\text { Texas A\&M University, and Paris } 6 \\
\text { pisier@math.tamu.edu }\end{array}$ & Tristan Rivière & $\begin{array}{l}\text { ETH, Switzerland } \\
\text { riviere@math.ethz.ch }\end{array}$ \\
\hline Igor Rodnianski & $\begin{array}{l}\text { Princeton University, USA } \\
\text { irod@math.princeton.edu }\end{array}$ & Wilhelm Schlag & $\begin{array}{l}\text { University of Chicago, USA } \\
\text { schlag@math.uchicago.edu }\end{array}$ \\
\hline Sylvia Serfaty & $\begin{array}{l}\text { New York University, USA } \\
\text { serfaty@ cims.nyu.edu }\end{array}$ & Yum-Tong Siu & $\begin{array}{l}\text { Harvard University, USA } \\
\text { siu@math.harvard.edu }\end{array}$ \\
\hline Terence Tao & $\begin{array}{l}\text { University of California, Los Angeles, USA } \\
\text { tao@math.ucla.edu }\end{array}$ & A Michael E. Taylor & $\begin{array}{l}\text { Univ. of North Carolina, Chapel Hill, USA } \\
\text { met@math.unc.edu }\end{array}$ \\
\hline Gunther Uhlmann & $\begin{array}{l}\text { University of Washington, USA } \\
\text { gunther@math.washington.edu }\end{array}$ & András Vasy & $\begin{array}{l}\text { Stanford University, USA } \\
\text { andras@math.stanford.edu }\end{array}$ \\
\hline Virgil Voiculescu & $\begin{array}{l}\text { University of California, Berkeley, USA } \\
\text { dvv@math.berkeley.edu }\end{array}$ & Steven Zelditch & $\begin{array}{l}\text { Northwestern University, USA } \\
\text { zelditch@math.northwestern.edu }\end{array}$ \\
\hline
\end{tabular}

\section{PRODUCTION}

contact@msp.org

Silvio Levy, Scientific Editor

Sheila Newbery, Senior Production Editor

See inside back cover or msp.berkeley.edu/apde for submission instructions.

The subscription price for 2012 is US \$140/year for the electronic version, and \$240/year for print and electronic. Subscriptions, requests for back issues from the last three years and changes of subscribers address should be sent to Mathematical Sciences Publishers, Department of Mathematics, University of California, Berkeley, CA 94720-3840, USA.

Analysis \& PDE, at Mathematical Sciences Publishers, Department of Mathematics, University of California, Berkeley, CA 94720-3840 is published continuously online. Periodical rate postage paid at Berkeley, CA 94704, and additional mailing offices.

APDE peer review and production are managed by EditFLOW ${ }^{\mathrm{TM}}$ from Mathematical Sciences Publishers.

PUBLISHED BY

mathematical sciences publishers

http://msp.org/

A NON-PROFIT CORPORATION

Typeset in IATEX

Copyright $(2012$ by Mathematical Sciences Publishers 


\section{ANALYSIS \& PDE}

\section{Volume $5 \quad$ No. $2 \quad 2012$}

The geodesic X-ray transform with fold caustics

Plamen STEFANOV and GUNTHER UHLMANN

Existence of extremals for a Fourier restriction inequality

Michael Christ and Shuanglin ShaO

Dispersion and controllability for the Schrödinger equation on negatively curved manifolds

NALINI ANANTHARAMAN and GABRIEL RIVIÈRE

A bilinear oscillatory integral estimate and bilinear refinements to Strichartz estimates on closed manifolds

\section{ZAHER HANI}

The Cauchy problem for the Benjamin-Ono equation in $L^{2}$ revisited

LUC MOLINET and DidIER PILOD

On triangles determined by subsets of the Euclidean plane, the associated bilinear operators 